ESAIM: COCV

Vol. 13, No 3,2007 , pp. 598-621

DOI: $10.1051 /$ cocv:2007037
ESAIM: Control, Optimisation and Calculus of Variations

www.edpsciences.org/cocv

\title{
MORSE INDEX AND BIFURCATION OF $p$-GEODESICS ON SEMI RIEMANNIAN MANIFOLDS
}

\author{
Monica Musso ${ }^{1,2}$, Jacobo Pejsachowicz ${ }^{1}$ and Alessandro Portaluri ${ }^{3}$
}

\begin{abstract}
Given a one-parameter family $\left\{g_{\lambda}: \lambda \in[a, b]\right\}$ of semi Riemannian metrics on an $n$ dimensional manifold $M$, a family of time-dependent potentials $\left\{V_{\lambda}: \lambda \in[a, b]\right\}$ and a family $\left\{\sigma_{\lambda}: \lambda \in\right.$ $[a, b]\}$ of trajectories connecting two points of the mechanical system defined by $\left(g_{\lambda}, V_{\lambda}\right)$, we show that there are trajectories bifurcating from the trivial branch $\sigma_{\lambda}$ if the generalized Morse indices $\mu\left(\sigma_{a}\right)$ and $\mu\left(\sigma_{b}\right)$ are different. If the data are analytic we obtain estimates for the number of bifurcation points on the branch and, in particular, for the number of strictly conjugate points along a trajectory using an explicit computation of the Morse index in the case of locally symmetric spaces and a comparison principle of Morse Schöenberg type.
\end{abstract}

Mathematics Subject Classification. 58E10, 37J45, 53C22, 58J30.

Received December 5, 2005. Revised January 19, 2006.

\section{INTRODUCTION}

Perturbed geodesics ( $p$-geodesics) are trajectories of a simple mechanical system on a semi Riemannian manifold $M$ of finite dimension $n$. They are critical points of the energy functional defined on the space of paths on $M$ by

$$
E(\gamma)=\int_{0}^{1} \frac{1}{2} g\left(\gamma^{\prime}(x), \gamma^{\prime}(x)\right) \mathrm{d} x-\int_{0}^{1} V(x, \gamma(x)) \mathrm{d} x
$$

where $g$ is a semi Riemannian metric on $M$ and $V$ is a time-dependent potential. When the metric is indefinite, the functional $E$ is of strongly indefinite type and the ordinary Morse index of its critical points is infinite. In [35], we associated to each non degenerate perturbed geodesic $\gamma$ a generalized Morse index $\mu_{\text {spec }}(\gamma) \in \mathbb{Z}$ and showed that it coincides with the conjugate index $\mu_{\mathrm{con}}(\gamma)$, an integer that counts algebraically the total number of conjugate points along $\gamma$. In the present paper we will use the generalized Morse index in order to study bifurcation of perturbed geodesics under various conditions including variation of metric and potential.

Recently, several kind of bifurcation phenomena have been studied in differential geometry and in mechanics. The change of qualitative properties of closed geodesics under one parameter variation of the metric was discussed

\footnotetext{
Keywords and phrases. Generalized Morse index, semi-Riemannian manifolds, perturbed geodesic, bifurcation.

1 Dipartimento di Matematica, Politecnico di Torino, Torino, Italy; jacobo.pejsachowicz@polito.it

2 Departamento de Matematicas, Pontificia Universidad Catolica de Chile, Avenida Vicuña MacKenna 4860, Macul, Chile; mmusso@mat.puc.cl

3 Departamento de Matemática, Instituto de Matemática e Estatística, Universidade de São Paulo, Rua do Matão 1010, CEP 05508-900, São Paulo, SP Brazil; portalur@ime.usp.br

Current address: Dipartimento di Matematica, Politecnico di Torino, Italy.
} 
in [29], Section 3.4, and $[7,10]$. How the crossing of 0 by a simple eigenvalue of the index form influences the topology of geodesics on a Lorentzian surface was studied in [6]. Bifurcation of relative equilibria of symmetric mechanical systems from a point of view close to ours was analyzed in [11]. A similar approach to bifurcation of minimal surfaces on a Riemannian manifold was taken in [26]. In this paper we will deal only with bifurcation of geodesics from a trivial branch. This is one of the typical bifurcation phenomena for solutions of differential equations studied in nonlinear functional analysis. Roughly speaking, given a smooth branch $\sigma \equiv\left\{\sigma_{\lambda}: \lambda \in[a, b]\right\}$ of perturbed geodesics, a bifurcation point from the trivial branch $\sigma$ is a point $\lambda_{*}$ in $[a, b]$ such that arbitrary close to the pair $\left(\lambda_{*}, \sigma_{\lambda_{*}}\right)$ there are pairs $\left(\lambda, \gamma_{\lambda}\right)$ where $\gamma_{\lambda}$ is a $p$-geodesic with the same end points as $\sigma_{\lambda}$ but not belonging to the branch $\sigma$.

If we take as trivial branch $\sigma_{\lambda}$ the restrictions of a given perturbed geodesic $\gamma:[0,1] \rightarrow M$ to subintervals of the form $[0, \lambda]$ it is easy to see that bifurcation can occur only at points which are conjugate to 0 .

Jacobi found a geometric characterization of conjugate points along extremals of a variational integral $\int_{0}^{1} L\left(x, y(x), y^{\prime}(x)\right) \mathrm{d} x$. He proved that conjugate points arise as intersection points of a given extremal with the envelope of the family of extremals through a point. By the envelope theory, this fact has as a consequence that all conjugate points along extremals of variational integrals are bifurcation points from the trivial branch. This was known from the very beginning of the calculus of variation (cf. [8], p. 63), although it was never formulated in terms of bifurcation simply because this theory was inexistent at that time. Jacobi's characterization extends to vector-valued variational integrals whose Lagrangian $L$ verifies the Legendre condition: $L_{\dot{q}_{i} \dot{q}_{j}}$ is positive definite. For example, in [22] this fact was used in order to give an alternate definition of conjugate point.

The energy functional of a Riemannian metric verifies the Legendre condition trivially. Therefore all conjugate points along perturbed geodesics on Riemannian manifold are bifurcation points (cf. Cor. 4.11, [29], Complement 2.1.13).

On semi Riemannian manifolds with indefinite metric the above conclusion does not hold anymore and conjugate points split in two classes: those which are points of bifurcation and those which are not. The present paper is devoted to the analysis of this situation. One of the consequences of the theory developed here is that, in the framework of semi Riemannian manifolds, bifurcation invariants become useful in order to find among all conjugate points those at which bifurcation occurs. We will call them strictly conjugate points here.

In general, conjugate points of perturbed geodesics need not to be isolated and when the metric is indefinite the same holds even in the geodesic case [25]. Because of this, we will adopt an approach to bifurcation invented by Krasnoselskij and his collaborators in the sixties [30]. This approach is based on topological invariants that are stable under perturbations and allows to deal with non isolated potential bifurcation points. An alternative non perturbative approach in the case of geodesics was explored in [24,39-41].

The methods are of functional analytic type. Although the book [29] is concerned with Riemannian manifolds only, it represents a main source for the analytic setting used here. The definition of the generalized Morse index in [35] is based on the concept of spectral flow of a family of Fredholm quadratic forms. It was shown in [19] that non vanishing of the spectral flow of the family of Hessians along the trivial branch leads to bifurcation of critical points of strongly indefinite functionals of Fredholm type. We will use an improved version of this result in order to prove our main theorem:

Let $M, g, V, \sigma$ be as described above. Assume that p-geodesics $\sigma_{a}$ and $\sigma_{b}$ are non degenerate (and hence have a well defined generalized Morse index).

If $\mu\left(\sigma_{a}\right) \neq \mu\left(\sigma_{b}\right)$, then there must be at least one point $\lambda_{*} \in(a, b)$ which is a bifurcation point from the trivial branch.

If $\sigma_{\lambda}$ is degenerate only at a finite number of points $\lambda \in I$, e.g. when all data are analytic, then there are at least $\left|\mu\left(\sigma_{a}\right)-\mu\left(\sigma_{b}\right)\right| / n$ distinct bifurcation points $\lambda \in(a, b)$.

In particular if the manifold and potential are analytic then along any non degenerate perturbed geodesic $\gamma$ there are at least $|\mu(\gamma)| / n$ strictly conjugate points.

The precise statement is in Section 4, Theorem 4.2, where we also derive other consequences regarding the number of strictly conjugate points along a perturbed geodesic. The proof of the theorem is deferred to Section 8 . 
When the manifold is a locally symmetric space, the contribution of the curvature tensor to the generalized Morse index can be computed quite explicitly, since the matrix of the curvature operator in a parallel orthonormal frame is constant.

Let $J$ be the canonical flat metric of index $\nu$ in $\mathbb{R}^{n}$. In Section 5 , for each symmetric matrix $S$ we define an index, analogous to the index for periodic solutions of linear autonomous Hamiltonian systems, considered in $[12,16,17,20,23]$, but associated to the Dirichlet boundary value problem for second order system $J u^{\prime \prime}+S u=0$. The index $\operatorname{ind}_{\mathrm{J}}(S)$ can be computed from the eigenvalues of the matrix $J S$ plus a correction term which can be easily estimated. The generalized Morse index of a geodesic on a locally symmetric space equals the index of the system arising from the equation for Jacobi fields written in a parallel orthonormal frame. Therefore, in Section 6, we take $\operatorname{ind}_{\mathrm{J}}(S)$ as our basic invariant and use it in Corollary 6.1 and Corollary 6.2 in order to obtain computable criteria for bifurcation and estimates for the number of bifurcation points along geodesics on locally symmetric spaces.

If the potential is time independent and the $p$-geodesic $\sigma_{a} \equiv p$ and $\sigma_{b} \equiv q$ are constant, then the number of bifurcation points along any trivial branch of perturbed geodesics with end points $\sigma_{a}$ and $\sigma_{b}$ can be estimated from below by the index of the Hessian of the potential $V$ at $p$ and $q$ respectively. This is the content of Corollary 6.3. On symmetric spaces this can be used in order to study bifurcation of periodic $p$-geodesics from an equilibrium point (cf. Cor. 6.4).

In a more general setting we can still obtain bounds from below for the number of bifurcation points using the previous calculations and a comparison principle. For geodesics this principle is an extension of the classical Morse-Schöenberg comparison theorem to semi Riemannian manifolds. For lightlike and timelike geodesics on Lorentzian manifolds it was proved earlier by Beem, Ehrlich and Easley [5].

The comparison principle is formulated in Section 7, Theorem 7.1 and proved in Section 9. In Section 7, we use Theorem 7.1 in several ways both for geodesics and for perturbed geodesics. In Corollaries 7.3 and 7.4 we estimate the number of strictly conjugate points along a geodesic on analytic semi Riemannian manifolds by comparison with a locally symmetric space of the same dimension and index. A similar approach leads to an estimate for the number of strictly conjugate points along a horizontal geodesic on a warped product with locally symmetric base in Corollary 7.5.

Finally, in Theorem 7.6 we obtain an estimate for the number of bifurcation points from a trivial branch of $p$-geodesics on a locally symmetric space by comparing the Morse index of a non degenerate $p$-geodesic with the Morse index of a geodesic with the same starting point.

The remaining sections are as follows: Section 2 contains a discussion of Jacobi's geometric characterization of conjugate points mentioned above. Section 3 is a short review of the results in [35] with the purpose to introduce the notations. In the appendix we sketch the proof of the vector bundle neighborhood theorem needed for the reduction of our problem to the framework considered in [19].

\section{Conjugate points and Bifurcation of EXtremals in CAlCulus of Variation}

As a motivation for the theory developed here, let us make a digression on the relation between the Jacobi's geometric characterization of conjugate points and bifurcation from the trivial branch.

Consider the one dimensional variational integral $\phi(y)=\int_{0}^{1} L\left(x, y, y^{\prime}\right) \mathrm{d} x$. Assume that $L$ is smooth with non vanishing partial derivative $\frac{\partial L}{\partial z}$. Assume moreover that the initial value problem $y(0)=0, y^{\prime}(0)=z$ for the Euler Lagrange equation of the functional $\phi$ has a solution $y(x, z)$ defined on the whole interval $[0,1]$.

The plane curve given by the graph $\gamma_{0}$ of the function $y_{0}(x)=y(x, 0)$ is naturally embedded into a one parameter family of curves $\gamma_{z}$, given by the graphs of $y_{z}(x)=y(x, z), 0 \leq x \leq 1$, passing through the point $(0,0)$.

The envelope $\mathcal{E}$ of this family of plane curves is by definition the set $\mathcal{E}=\left\{(x, y) \mid y-y(x, z)=0, \frac{\partial y}{\partial z}(x, z)=0\right\}$. Jacobi characterized the points of the extremal $\gamma_{0}$ conjugate to $\gamma_{0}(0)$ as intersection points of $\gamma_{0}$ with $\mathcal{E}$. Since $\mathcal{E}$ can be also seen as the set of limit points of intersections of nearby curves in the family (the set of intersections points of two consecutive curves, in the pictoresque terminology of G. Peano [38]), Jacobi's characterization leads to the following conclusion ( $c f .[8]$, p. 63): 
Proposition 2.1. If the point $Q_{*}=\gamma_{0}\left(x_{*}\right)$ is conjugate to $\gamma_{0}(0)$ along $\gamma_{0}$, then for small enough $z$ the extremal $\gamma_{z}$ intersects $\gamma_{0}$ at a unique point $Q_{z}=\gamma_{0}\left(x_{z}\right)$ such that $Q_{z} \rightarrow Q_{*}$ as $z \rightarrow 0$.

The proof of Proposition 2.1 is quite simple. It can be reduced to a very special case of one of the basic results in bifurcation theory, known as Crandal-Rabinowitz bifurcation theorem. For this, let us consider the variable $x$ as a parameter. Let $f:[0,1] \times \mathbb{R} \rightarrow \mathbb{R}$ be defined by $f(x, z)=y(x, z)-y(x, 0)$. Then the equation $f(x, z)=0$ has a trivial branch of solutions of the form $(x, 0)$. Since $Q_{*} \in \mathcal{E}$, we have that $\frac{\partial f}{\partial z}\left(x_{*}, 0\right)=\frac{\partial y}{\partial z}\left(x_{*}, 0\right)=0$. On the other hand, $\frac{\partial y}{\partial z}(x, 0)$ is a Jacobi field along $y_{0}$ derived from a nontrivial deformation of $y_{0}$ by extremals. Since $\frac{\partial y}{\partial z}\left(x_{*}, 0\right)=0$, the partial $\frac{\partial^{2} y}{\partial x \partial z}\left(x_{*}, 0\right)=\frac{\partial^{2} f}{\partial x \partial z}\left(x_{*}, 0\right)$ cannot vanish. Thus, we are in the hypothesis of the Crandal-Rabinowitz theorem [15]. Under this hypothesis a nontrivial branch of solutions of $f(x, z)=0$ of the form $(x(z), z)=0$ with $x(0)=x_{*}$ can be found by developing in Taylor series the rescaled function $g(w)=f\left(x_{*}+z w, z\right)$ dividing by $z^{2}$ and solving in $w$ using the implicit function theorem. Then the points $Q_{z}=(x(z), z)$ are intersection points of $\gamma_{z}$ with $\gamma_{0}$ such that $Q_{z} \rightarrow Q_{*}$ as $z \rightarrow 0$.

Of course, the relation of the envelope of a family of curves with bifurcation, understood in the broad sense, is not limited to the variational setting. It is one of the main subjects of singularity theory.

The approach to bifurcation of perturbed geodesics which we will use in this paper is a reinterpretation of Proposition 2.1 from the point of view of nonlinear functional analysis. Let us explain this using our example.

We look for solutions of a parametrized family of boundary value problems for the Euler-Lagrange equations of the variational integral that are close to the restrictions of our distinguished solution $y_{0}$ to subintervals $[0, \lambda]$, but in order to work in a fixed space of functions, we reparametrize them. To be more precise, we choose a $\delta>0$ such that on $\gamma_{0}([0, \delta])$ there are no conjugate points to $Q_{0}=(0,0)$. Then, for $\lambda \in[\delta, 1]$, we consider $\tilde{y}_{\lambda}(x)=y(\lambda \cdot x, 0)$. The path $\tilde{y}:[\delta, 1] \rightarrow C^{2}[0,1]$ defined by $\tilde{y}(\lambda)=\tilde{y}_{\lambda}$ will be considered as a trivial branch of solutions of the family of boundary value problems

$$
\frac{\mathrm{d}}{\mathrm{d} x} L_{u^{\prime}}\left(\lambda, x, u, u^{\prime}\right)-L_{u}\left(\lambda, x, u, u^{\prime}\right)=0, u(0)=0, u(1)=y_{0}(\lambda)
$$

where $L(\lambda, x, y, z)=L(\lambda x, y, 1 / \lambda z)$.

If in Proposition 2.1 we take $z_{n}=1 / n, \lambda_{n}=x_{z_{n}}$ and $y_{n}(x)=y\left(\lambda_{n} \cdot x, z_{n}\right)$, then we have that $\lambda_{n} \rightarrow x_{*}$, the sequence $y_{n}$ converges to $\tilde{y}\left(x_{*}\right)$ in $C^{2}[0,1]$, and each $y_{n}$ is a solution of $(2.1)$ not in the branch $\tilde{y}$. Thus, by the very definition of bifurcation point, any conjugate instant $\lambda=x_{*}$ along $y_{0}$ is a bifurcation point for solutions of (2.1) from the trivial branch $\tilde{y}$.

In the particular case considered above a whole smooth curve of nontrivial solutions $y_{z}(x)=y\left(x_{z} x, z\right)$ branches of from $\tilde{y}$ at $\tilde{y}\left(x_{*}\right)$. However under the general topological assumptions of our main theorem we will only obtain a sequence of nontrivial solutions converging to the trivial branch.

\section{Perturbed geodesics And the EnERgy functional}

Let $M$ be a smooth connected manifold of dimension $n$. Let $g$ be a semi Riemannian metric on $M$ and let $V:[0,1] \times M \rightarrow \mathbb{R}$ be a smooth time-dependent potential. We will denote by $D$ the associated Levi-Civita connection and by $D \mathrm{~d} x$ the covariant derivative of a vector field along a smooth curve $\gamma$. Here we use the notation introduced by Milnor, in [34].

A perturbed geodesic (p-geodesic) is a solution $\gamma:[0,1] \rightarrow M$ of the second order differential equation

$$
\frac{D}{\mathrm{~d} x} \gamma^{\prime}(x)+\nabla V(x, \gamma(x))=0
$$

where $\nabla$ is the gradient of a function on $M$ with respect to the metric $g$.

Let $\Omega$ be the manifold of all $H^{1}$-paths in $M$. It is well known that $\Omega$ is a smooth Hilbert manifold modelled by $H^{1}\left([0,1] ; \mathbb{R}^{n}\right)$. The tangent space $T_{\gamma} \Omega$ at the point $\gamma$ can be identified in a natural way with the Hilbert space 
$H^{1}(\gamma)=\left\{\xi \in H^{1}([0,1] ; T M): \tau \xi=\gamma\right\}$ of all $H^{1}$-vector fields along $\gamma$. Here $\tau: T M \rightarrow M$ is the projection of the tangent bundle of $M$ to its base.

Any choice of a Riemannian (positive definite) metric on $M$ endows $\Omega$ with an associated Riemannian structure and hence with a distance which makes $\Omega$ a metric space. The end-point map

$$
\pi: \Omega \rightarrow M \times M ; \quad \pi(\gamma)=(\gamma(0), \gamma(1))
$$

is known to be a submersion and therefore for each $(p, q) \in M \times M$ the fiber

$$
\Omega_{p, q}=\{\gamma \in \Omega: \gamma(0)=p, \gamma(1)=q\}
$$

is a sub-manifold of codimension $2 n$ whose tangent space $T_{\gamma} \Omega_{p, q}$ is the subspace $H_{0}^{1}(\gamma)$ of $H^{1}(\gamma)$ given by

$$
H_{0}^{1}(\gamma)=\left\{\xi \in H^{1}(\gamma): \xi(0)=\xi(1)=0\right\} .
$$

Since $\pi$ is a submersion, the family of Hilbert spaces $H_{0}^{1}(\gamma)$ is a Hilbert bundle $T F(\pi)=$ ker $T \pi$ over $\Omega$, called the bundle of tangents along the fibers. The reference for all the above, and for everything else in this section is [35].

Associated to each pair $(g, V)$ there is an energy functional $\bar{E}: \Omega \rightarrow \mathbb{R}$ defined by

$$
\bar{E}(\gamma)=\int_{0}^{1} \frac{1}{2} g\left(\gamma^{\prime}(x), \gamma^{\prime}(x)\right) \mathrm{d} x-\int_{0}^{1} V(x, \gamma(x)) \mathrm{d} x .
$$

The critical points of the restriction $E=E_{p, q}$ of $\bar{E}$ to the sub-manifold $\Omega_{p, q}$ are precisely the $p$-geodesics through $p, q$ with kinetic energy $\frac{1}{2} g\left(\gamma^{\prime}, \gamma^{\prime}\right)$ and potential energy $V$. To be more precise, critical points of the energy functional are weak solutions of (3.1), which turn out to be smooth classical solutions by elliptic regularity.

In [35], we associated two integers to each non degenerate critical point of the energy functional. The first integer is the generalized Morse index $\mu_{\text {spec }}(\gamma)$, defined as the negative of the spectral flow of the Hessians of the energy functionals along the path $\tilde{\gamma}$ canonically induced by $\gamma$ on $\Omega$. Since several points related to the construction of the generalized Morse index will be used in the proof of our main theorem, we will briefly recall the construction here.

The linearization of the boundary value problem

$$
\left\{\begin{array}{l}
\frac{D}{\mathrm{~d} x} \gamma^{\prime}(x)+\nabla V(x, \gamma(x))=0 \\
\gamma(0)=p, \quad \gamma(1)=q
\end{array}\right.
$$

at the critical point $\gamma$ is the equation of Jacobi fields

$$
\frac{D^{2}}{\mathrm{~d} t} \xi(t)+R\left(\gamma^{\prime}(t), \xi(t)\right) \gamma^{\prime}(t)+D_{\xi(t)} \nabla V(t, \gamma(t))=0,
$$

subjected to Dirichlet boundary conditions $\xi(0)=0=\xi(1)$. Here $\xi$ is a vector field along $\gamma, R$ is the curvature tensor of the connection $D$ and $D_{\xi(x)} \nabla V(x, \gamma(x))$ is the Hessian of $V(x,-)$ with respect to the metric $g$, viewed as a symmetric operator of $T_{\gamma(x)} M$.

Its weak solutions $\left(H_{0}^{1}\right.$-Jacobi fields) are vector fields $\xi \in H_{0}^{1}(\gamma)$ such that for any $\eta \in H_{0}^{1}(\gamma)$

$$
0=H_{\gamma}(\xi, \eta)=\int_{0}^{1} g\left(\frac{D}{\mathrm{~d} x} \xi(x), \frac{D}{\mathrm{~d} x} \eta(x)\right) \mathrm{d} x
$$

$$
-\int_{0}^{1} g\left(R\left(\gamma^{\prime}(x), \xi(x)\right) \gamma^{\prime}(x)+D_{\xi(x)} \nabla V(x, \gamma(x)), \eta(x)\right) \mathrm{d} x .
$$


Thus, Jacobi fields in $H_{0}^{1}(\gamma)$ are the elements of the kernel of the bilinear form $H_{\gamma}$. The Hessian of $E_{p, q}$ at $\gamma$ is the quadratic form $h_{\gamma}: H_{0}^{1}(\gamma) \rightarrow \mathbb{R}$ associated to $H_{\gamma}$.

Since the embedding of $H_{0}^{1}(\gamma)$ into $L^{2}(\gamma)$ is compact, the form

$$
c(\xi)=\int_{0}^{1} g_{\lambda}\left(R\left(\gamma^{\prime}(x), \xi(x)\right) \gamma^{\prime}(x)+D_{\xi(x)} \nabla V(x, \gamma(x)), \xi(x)\right) \mathrm{d} x
$$

is weakly semi-continuous. Being a weakly semi-continuous perturbation of a non degenerate form, $h_{\gamma}$ is a bounded Fredholm quadratic form. In particular $\operatorname{ker} h_{\gamma} \equiv\left\{\xi \mid H_{\gamma}(\xi, \eta)=0\right.$ for all $\left.\eta\right\}$ is finite dimensional.

The form $h_{\gamma}$ is non degenerate if and only if the instant 1 is not conjugate to 0 along $\gamma$. If this holds we will say that the $p$-geodesic is non degenerate.

Each $p$-geodesic $\gamma$ induces in a canonical way a path $\tilde{\gamma}$ on the manifold $\Omega$ of all paths on $M$. The canonical path $\tilde{\gamma}:[0,1] \rightarrow \Omega$ associated to $\gamma$ is defined by $\tilde{\gamma}(\lambda)(x)=\gamma(\lambda \cdot x), x \in[0,1]$. Each $\tilde{\gamma}_{\lambda} \equiv \tilde{\gamma}(\lambda)$ is a $p$-geodesic for $\left(g, V_{\lambda}\right)$, where $V_{\lambda}(x, m)=\lambda^{2} V(\lambda x, m)$. From the above discussion it follows that the Hessian $h_{\lambda}$ of $E_{\lambda} \equiv E_{p, \gamma(\lambda)}$ at $\tilde{\gamma}_{\lambda}$ is degenerate if and only $\gamma(\lambda)$ is a conjugate point to $p=\gamma(0)$ along $\gamma$. If $\gamma$ is non degenerate the path $\tilde{\gamma}$ has non degenerate end points. The family $h_{\lambda}, \lambda \in[0,1]$ defines a function $h$ on the total space of the pull-back bundle $\tilde{\gamma}^{*}(T F)$ over $[0,1]$ such that its restriction to each fiber is a bounded quadratic form and such that $h_{0}, h_{1}$ are non degenerate. This is what we called in [35] an admissible family of Fredholm quadratic forms. The spectral flow of such a family was defined in [35], Section 2.

Roughly speaking the spectral flow is a measure of how much of a negative subspace for $h_{\lambda}$ at $\lambda=0$ becomes positive at $\lambda=1$ minus the part of a positive subspace that becomes negative. In general the spectral flow depends on the homotopy class of the path of quadratic forms but in this specific case it depends only on the end points. This allows to define a relative form of Morse index even in the case when the classical Morse indices are infinite. By definition the generalized Morse index of a $p$-geodesic $\gamma$ is

$$
\mu_{\mathrm{spec}}(\gamma)=-\operatorname{sf}(h)
$$

The second integer, $\mu_{\mathrm{con}}(\gamma)$, called conjugate index, was defined using Brouwer degree in order to count zeroes of a determinant associated to the Jacobi equation by means of trivalization via a parallel orthonormal frame (see [35], Sect. 4, for a detailed description).

Finally, we proved a generalization of the Morse Index Theorem to perturbed geodesics on semi Riemannian manifolds [35], Theorem 1.1, by establishing the equality

$$
\mu_{\text {spec }}(\gamma)=\mu_{\text {con }}(\gamma)
$$

Taking into account (3.5) we will denote both indices with $\mu(\gamma)$.

\section{The MAIn THEOREM AND SOME CONSEQUENCES}

Let $p: \mathcal{S}_{\nu}^{2}(M) \rightarrow M$ be the bundle of all non degenerate symmetric two forms of index $\nu$ on the tangent bundle $T M$. Let $I=[a, b]$ be an interval. A one parameter family of semi Riemannian metrics on $M$ is a smooth map $g: I \times M \rightarrow \mathcal{S}_{\nu}^{2}(M)$ such that $p g=\pi$, where $\pi$ denotes the projection onto the second factor.

Each $g_{\lambda}=g(\lambda,-)$ is then a semi Riemannian metric on $M$ of index $\nu$. Using the Koszul formula it is easy to see that the family of associated Levi-Civita connections $D^{\lambda}$ is smooth with respect to the $\lambda$ variable in an obvious sense.

If $V: I \times[0,1] \times M \rightarrow \mathbb{R}$ is a smooth function, we consider $V$ as a smooth one parameter family of timedependent potentials $V_{\lambda}:[0,1] \times M \rightarrow \mathbb{R}$ defined by $V_{\lambda}(x, u)=V(\lambda, x, u)$. The data $\left(M, g_{\lambda}, V_{\lambda}\right)_{\lambda \in I}$ define a one parameter family of time-dependent mechanical systems.

We will also assume the existence of a one parameter family of known $p$-geodesics. In other words, given a smooth family $\left(g_{\lambda}, V_{\lambda}\right)_{\lambda \in I}$ as above, we assume that there exists a smooth map $\sigma:[a, b] \times[0,1] \rightarrow M$ such that for each $\lambda \in I, \sigma_{\lambda}(x) \equiv \sigma(\lambda, x)$ is a $p$-geodesic corresponding to the mechanical system $\left(g_{\lambda}, V_{\lambda}\right)$. 
We will refer to the family $\left(\sigma_{\lambda}\right)_{\lambda \in[a, b]}$ as the trivial branch of perturbed geodesics.

Definition 4.1. A point $\lambda_{*}$ is called a bifurcation point for $p$-geodesics from the trivial branch $\sigma$ if there exists a sequence $\left(\lambda_{n}, \gamma_{n}\right) \rightarrow\left(\lambda_{*}, \sigma\left(\lambda_{*}\right)\right)$ in $I \times \Omega$ such that $\gamma_{n}$ is a weak solution of equation (3.1) with boundary conditions

such that $\gamma_{n}$ does not belong to $\sigma(I)$.

$$
\gamma_{n}(0)=\sigma_{\lambda_{n}}(0), \quad \gamma_{n}(1)=\sigma_{\lambda_{n}}(1)
$$

As stated above the notion of bifurcation point depends on the metric induced on $\Omega$ by an arbitrary choice of Riemannian metric on $M$. As a matter of fact using a slightly weaker topology the concept of bifurcation point can be defined without appealing to the existence of a Riemannian metric on $M$. There is an embedding of $\Omega$ into the space of continuous paths in $M$ endowed with the compact-open topology (see for example [28], Lem. 2.4.6). Thus, the sequence $\gamma_{n}$ in the above definition converges to $\sigma\left(\lambda_{*}\right)$ in $C^{0}([0,1] ; M)$ as well. A metric free definition of bifurcation point would be as follows: it is a point such that any neighborhood of $\left(\lambda_{*}, \sigma\left(\lambda_{*}\right)\right)$ in $I \times C^{0}([0,1] ; M)$ contains a solution of the boundary value problem (3.1), (4.1) which is not in the trivial branch. Finally notice that, by bootstrap, $\gamma_{n}$ converges to $\sigma\left(\lambda_{*}\right)$ in the Whitney topology of $C^{\infty}(I ; M)$. Therefore, the choice of any of the above approaches gives the same bifurcation points.

For each $\lambda \in I$, let $E_{\lambda}$ be the restriction of the energy functional associated to the data $\left(g_{\lambda}, V_{\lambda}\right)$ to the sub-manifold $\Omega_{\lambda} \equiv \Omega_{\sigma_{\lambda}(0), \sigma_{\lambda}(1)}$. The trivial branch $\sigma$ can be viewed as a smooth path $\sigma: I \rightarrow \Omega$ of critical points $\sigma(\lambda)=\sigma_{\lambda}$ of $E_{\lambda}$.

Our main result is the following theorem.

Theorem 4.2. Let $M$ and $\left(g_{\lambda}, V_{\lambda}\right)_{\lambda \in I}$ be as above. Let $\sigma$ be a trivial branch of $p$-geodesics of $\left(M, g_{\lambda}, V_{\lambda}\right)_{\lambda \in I}$ such that the end points $\sigma_{a}$ and $\sigma_{b}$ are non degenerate.

(i) If $\mu\left(\sigma_{a}\right) \neq \mu\left(\sigma_{b}\right)$, then there exists at least one bifurcation point, $\lambda_{*} \in(a, b)$, from the trivial branch.

(ii) If $\sigma_{\lambda}$ is degenerate only at a finite number of points $\lambda \in I$ then there are at least $\left|\mu\left(\sigma_{a}\right)-\mu\left(\sigma_{b}\right)\right| / n$ distinct bifurcation points $\lambda$ in $(a, b)$.

Remark 4.3. The hypothesis in part (ii) of Theorem 4.2 is automatically verified in the following two cases:

(a) The data $(M, g, V, \sigma)$ are analytic. In this case the singular set $\Sigma=\left\{\lambda \in I \mid \operatorname{ker} h_{\lambda} \neq 0\right\}$ can be characterized as the set of zeroes of an analytic "determinant" function [18]. Since $h_{a}$ is non degenerate this function does not vanish on the whole interval $I$ and hence $\Sigma$ must be discrete.

(b) The intrinsic derivative (see Sect. 8) of the generalized family $h_{\lambda}, \lambda \in I$ of Hessians at any point $\lambda_{*} \in \Sigma$ is a non degenerate quadratic form.

Remark 4.4. In the estimate of Theorem 4.2 the dimension $n$ of the manifold arises because it provides a bound for the possible dimensions of the space of $H_{0}^{1}$-Jacobi fields. The estimate can be slightly improved for geodesics $(V \equiv 0)$. A $H_{0}^{1}$-Jacobi field along a geodesic $\gamma$ must be perpendicular to $\gamma^{\prime}(x)$ at any point $x \in[0,1]$, which implies that the dimension of the space of Jacobi fields vanishing at the end points cannot be greater than $(n-1)$. Therefore in the case of a geodesic we can substitute $n$ with $(n-1)$ in Theorem 4.2.

As already mentioned in the introduction, we will pay special attention to the following particular case: We will take $g$ and the potential $V$ fixed, take a $p$-geodesic $\gamma:[0,1] \rightarrow M$ and consider as the trivial branch the path $\tilde{\gamma}:[0,1] \rightarrow \Omega$ canonically induced by $\gamma$.

Definition 4.5. A conjugate point $\gamma\left(\lambda_{*}\right), \lambda_{*} \in[0,1]$, will be called a strictly conjugate if $\lambda_{*}$ is a bifurcation point from the trivial branch $\tilde{\gamma}$ in the sense of Definition 4.1 .

Not all conjugate points are strictly conjugate. Here is an example of a $p$-geodesic on a 3-dimensional semi Riemannian manifold with an isolated conjugate point which is not strictly conjugate.

Example 4.6. Let us consider the manifold $M=\mathbb{R}^{3}$ endowed with the metric $g_{0}=\mathrm{d} x^{2}-\mathrm{d} y^{2}+\mathrm{d} z^{2}$ and take as potential the function $V(x, y, z)=1 / 2 x^{2}-1 / 2 y^{2}+1 / 3 x^{3} y^{3}$. Since the gradient of $V$ with respect to $g$ is 
$\nabla V(z, y, z)=\left(x+x^{2} y^{3}\right) \frac{\partial}{\partial x}+\left(y-x^{3} y^{2}\right) \frac{\partial}{\partial y}$, the equations of a $p$-geodesic are

$$
\begin{cases}x^{\prime \prime}+x+x^{2} y^{3} & =0 \\ y^{\prime \prime}+y-x^{3} y^{2} & =0 \\ z^{\prime \prime} & =0\end{cases}
$$

In order to simplify the coefficients we will not normalize the interval: we consider the $p$-geodesic $\gamma_{0}(t)=(0,0, t)$ defined on $[0,2 \pi]$, connecting $(0,0,0)$ to $(0,0,2 \pi)$. We claim that there are no $p$-geodesics $\gamma \neq \gamma_{0}$ such that $\gamma(0)=\gamma_{0}(0)$ and $\gamma(\lambda)=\gamma_{0}(\lambda)$ for $\lambda \in[0,2 \pi]$.

Indeed, if $\gamma(t)=(x(t), y(t), z(t))$ verifies (4.2) and the above boundary conditions, then multiplying the first equation of (4.2) by $y(t)$, the second by $x(t)$, substracting the second from the first and integrating from 0 to $\lambda$ we obtain $\int_{0}^{\lambda}\left(x^{2} y^{4}+x^{4} y^{2}\right) d t=0$. Thus both $x, y \equiv 0$ and $\gamma(t)=\gamma_{0}(t)$.

On the other hand there is a two dimensional subspace of Jacobi fields along $\gamma_{0}, \xi(t)=a \sin t \frac{\partial}{\partial x}+b \sin t \frac{\partial}{\partial y}$ verifying the Dirichlet boundary conditions $\xi(0)=0=\xi(\pi)$. Therefore $\lambda=\pi$ is a conjugate point which is not strictly conjugate.

In order now to construct an example of a geodesic with a conjugate point which is not strictly conjugate we will reduce the problem to the previous one using the well known correspondence between $p$-geodesics and geodesics of the associated Jacobi metric together with a construction in [25] of a geodesic with assigned curvature operator on the normal space.

Example 4.7. Let $M$ and $g_{0}$ be as in the previous example and let us consider the conformally flat metric $g=\exp (2 \rho) g_{0}$, where $\rho$ is defined by

$$
\rho(x, y, z)=1 / 2 y^{2}-1 / 2 x^{2}+1 / 3 x^{3} y^{3} .
$$

The Levi Civita connexions $D$ of $g$ and $D^{0}$ of $g_{0}$ are related by

$$
D_{X} Y=D_{X}^{0} Y+d \rho(X) Y+d \rho(Y) X-g_{0}(X, Y) \nabla^{0} \rho
$$

where $\nabla^{0}$ is the gradient with respect $g_{0}$.

From this it follows easily that $\gamma_{0}(t)=(0,0, \sqrt{2} t)$ is a geodesic with energy $\mathcal{E}\left(\gamma_{0}\right)=1 / 2 g\left(\gamma^{\prime}(t), \gamma^{\prime}(t)\right)=1$. Moreover the vector fields $\frac{\partial}{\partial x}, \frac{\partial}{\partial y}$ are parallel along $\gamma_{0}$ and generate the normal space $T_{\gamma(t)}^{\perp} M$. Calculating the curvature tensor we get $R\left(\frac{\partial}{\partial z}, \frac{\partial}{\partial x}\right) \frac{\partial}{\partial z}=\frac{\partial}{\partial x}$ and $R\left(\frac{\partial}{\partial z}, \frac{\partial}{\partial y}\right) \frac{\partial}{\partial z}=\frac{\partial}{\partial y}$. Therefore the Jacobi equations for a normal vector field $\xi(t)=u(t) \frac{\partial}{\partial x}+v(t) \frac{\partial}{\partial y}$ are

$$
\left\{\begin{array}{l}
u^{\prime \prime}+u=0 \\
v^{\prime \prime}+v=0
\end{array}\right.
$$

As before, the Dirichlet problem $\xi(0)=0=\xi(\pi)$ for (4.4) has a two dimensional subspace of nontrivial solutions and therefore $\pi$ is a conjugate instant. However we will show that there cannot be non trivial geodesics bifurcating from the trivial branch $\gamma_{0}$.

Assume that $\gamma(t)=(x(t), y(t), z(t))$ is a geodesic for $g$ with $\gamma(0)=(0,0,0)$ and $\gamma(b)=(0,0, \lambda)$. If $\gamma$ is close enough to $\gamma_{0}$ then is $\gamma$ is spacelike and, possibly after changing the interval of definition, we can assume that the energy $\mathcal{E}(\gamma)=1$. By Jacobi's theorem [1], Theorem 3.7.7, geodesics for $g$ with energy 1 are reparametrizations of the perturbed geodesics for the mechanical system $\left(M, g_{0},-\exp (2 \rho)\right)$ with total energy $\mathcal{E}=0$.

The equations for the corresponding $p$-geodesic are

$$
\begin{cases}x^{\prime \prime}+2 \exp 2 \rho\left(x-x^{2} y^{3}\right) & =0 \\ y^{\prime \prime}+2 \exp 2 \rho\left(y+x^{3} y^{2}\right) & =0 \\ z^{\prime \prime} & =0\end{cases}
$$


If $\delta(t)=\gamma(h(t))$ verifies the above equations then multiplying the first equation of (4.5) by $y(t)$, the second by $x(t)$ substracting the second from the first as in the previous example and then integrating from 0 to $t_{*}=h^{-1}(\lambda)$ we obtain that $x \equiv 0 \equiv y$ and hence $\gamma=\gamma_{0}$.

We will now discuss some consequences of Theorem 4.2.

Corollary 4.8. Any regular p-geodesic $\gamma$ with non-vanishing index has a strictly conjugate point. If the data are analytic there must be at least $|\mu(\gamma)| / n$ of them.

For example, assume that $(M, g)$ is complete, take a point $p \in M$ and consider the exponential map $\exp _{p}: T_{p}(M) \rightarrow M$. It is well known that points conjugate to $p$ along $\gamma(x)=\exp _{p}(x \cdot v)$ are the critical values of the exponential map $\exp _{p}$ lying on $\gamma$. Consider

$$
B_{p}=\left\{m \in M \mid \text { for some } v \in \exp ^{-1}(m) \text {, exp is not locally one to one at } v\right\} .
$$

When $M$ is a Riemannian manifold, $B_{p}$ coincides with the set $C_{p}$ of all critical values of the exponential map (see [29], Th. 2.1.12). On general semi Riemannian manifolds, $B_{p}$ is only a closed proper subset of $C_{p}$. However, by definition of bifurcation point, the exponential $\exp _{p}$ cannot be a local diffeomorphism at any point of the form $t_{*} \gamma^{\prime}(0)$ if $t_{*}$ is a strictly conjugate instant. Therefore by the previous corollary any non degenerate geodesic $\gamma$ starting from $p$ crosses $B_{p}$ in at least $|\mu(\gamma)| /(n-1)$ points.

In the remaining part of this section we will discuss some criteria for isolated conjugate points to be strictly conjugate.

Let $\mathcal{I}$ be the $n$-dimensional vector space of all Jacobi fields along $\gamma$ verifying $\xi(0)=0$. Consider $\mathcal{I}[x]=\{\xi(x)$ : $\xi \in \mathcal{I}\} \subset T_{\gamma(x)} M$. It is easy to see that $\gamma\left(\lambda_{*}\right), \lambda_{*} \in[0,1]$, is conjugate to 0 if and only if $\mathcal{I}\left[\lambda_{*}\right] \neq T_{\gamma\left(\lambda_{*}\right)} M$. Let us denote by $\mathcal{I}[x]^{\perp}$ the $g$ orthogonal of $\mathcal{I}[x]$.

The conjugate instant $\lambda_{*}$ is said to be regular if the quadratic form $\Gamma\left(\lambda_{*}\right)$ given by the restriction of $g_{\gamma\left(\lambda_{*}\right)}$ to $\mathcal{I}\left[\lambda_{*}\right]^{\perp}$ is non degenerate. It is shown in [35], that regular conjugate instants are isolated and moreover the variation of the generalized Morse index through $\lambda^{*}$ is given by the signature of the quadratic form $\Gamma\left(\lambda_{*}\right)$. More precisely, for $\epsilon$ small enough,

From the main theorem it follows

$$
\mu\left(\tilde{\gamma}_{\lambda_{*}+\epsilon}\right)-\mu\left(\tilde{\gamma}_{\lambda_{*}-\epsilon}\right)=\operatorname{sign} \Gamma\left(\lambda_{*}\right) .
$$

Corollary 4.9. Any regular conjugate point such that $\operatorname{sign}\left(\left.g\right|_{\left.\mathcal{L}_{\left[\lambda_{*}\right]^{\perp}}\right)} \neq 0\right.$ is strictly conjugate.

Remark 4.10. The conjugate points in the previous examples are both non degenerate but with vanishing signature.

If $g$ is positive definite then any conjugate point is regular and the signature of the form $\Gamma\left(\lambda_{*}\right)$ is simply the dimension of $\mathcal{I}\left[\lambda_{*}\right]^{\perp}$. Therefore:

Corollary 4.11. If $(M, g, V)$ is a mechanical system on a Riemannian manifold all conjugate points along a p-geodesic $\gamma$ are strictly conjugate.

When $\lambda_{*}$ is not regular but yet isolated then its local contribution $\mu_{\mathrm{con}}\left(\lambda_{*}\right)$ to the variation of the Morse index can be still defined as in [35], (4.14). Thus $\lambda_{*}$ is a bifurcation point whenever $\mu_{\text {con }}\left(\lambda_{*}\right) \neq 0$. The local invariant $\mu_{\mathrm{con}}\left(\lambda_{*}\right)$ can be computed either by the algorithm for computing the degree of an analytic plane vector field as described in [35], Lemma 4.3, or using the calculation of $\mu_{\text {Maslov }}\left(\lambda_{*}\right)$ in the degenerate case via partial signatures as in $[24,42]$. However neither the algorithm for the degree nor the formula by partial signatures can be considered effective. This is one of the motivations for the approach adopted in this paper.

\section{Computation of the generalized Morse index}

In this section we will consider some special cases in which the generalized Morse index can be found explicitly, providing computable criteria for the existence of bifurcation points. As motivation, let us discuss first the Morse index of a geodesic on a Riemannian locally symmetric space. 
If $\gamma:[0,1] \rightarrow M$ is a geodesic, then, by Corollary 4.11 , the conjugate instants are isolated and each one of them gives a strictly positive contribution $m(\lambda)=\operatorname{dim} \operatorname{ker} h_{\lambda}$ to the index $\mu(\gamma)$. The index of a geodesic is not easy to compute in general but when the related second order system has constant coefficients one can easily get explicit formulas.

For example, if $M$ has a constant positive sectional curvature $K>0$ then the conjugate points correspond to instants $\lambda_{k}=k \pi / L \sqrt{K}$, where $L$ is the length of the geodesic $\gamma$. Moreover the multiplicity of each conjugate point is $(n-1)$. Thus $\gamma$ is non degenerate if $L \sqrt{K} / \pi$ is not an integer and its index is given by $\mu(\gamma)=(n-1) N_{K}(\gamma)$, where $N_{K}(\gamma)=[L \sqrt{K} / \pi]$ is the integral part of $L \sqrt{K} / \pi$. One can use this in Theorem 4.2 in order to estimate the number of geodesics bifurcating from a known trivial branch $\sigma_{\lambda}$. Assume, for instance, that there exists a smooth family $g_{\lambda}$ of metrics on $M$ connecting two metrics of constant positive sectional curvature $K, K^{\prime}$. If $\sigma:[a, b] \rightarrow \Omega(M)$ is a path such that each $\sigma_{\lambda}$ is geodesic for the metric $g_{\lambda}$ and $\sigma_{a}, \sigma_{b}$ are non degenerate, then by Theorem 4.2 there are at least $\left|N_{K}\left(\sigma_{a}\right)-N_{K^{\prime}}\left(\sigma_{b}\right)\right|$ bifurcation points from the trivial branch $\sigma$. In particular, if $g$ is a fixed metric of constant positive curvature then along any branch of geodesics with a large enough variation of length there will be bifurcation points from the branch. All of the above extends immediately to geodesics on locally symmetric spaces by considering the eigenvalues of the matrix of the curvature operator $R\left(\gamma^{\prime}, v\right) \gamma^{\prime}$ in a parallel orthonormal frame.

The generalized Morse index for $p$-geodesics on a semi Riemannian manifold constructed in [35] is not always positive and hence it is a considerable weaker object than the classical Morse index. Even in the case of locally symmetric spaces $(D R \equiv 0)$ formulas for the index are harder to compute than in the Riemannian case. Nevertheless, the above topological picture is still present in this context and leads to estimates for the number of bifurcation points.

Let $\gamma:[0,1] \rightarrow M$ be a $p$-geodesic on $M$ for $(g, V)$, with $g$ a semi Riemannian metric, and let $\gamma_{\lambda}(x)=\gamma(\lambda x)$, $\lambda \in[0,1]$. Choosing a parallel $g$-orthonormal frame $\mathcal{F}=\left\{e^{i}(x): i=1, \ldots, n\right\}$ along $\gamma$ and trivializing $\gamma_{\lambda}^{*}(T M)$ by means of the frame $\mathcal{F}_{\lambda}=\left\{e^{i}(\lambda x): i=1, \ldots, n\right\}$, by the computation performed in [35], Section 4 we have that such a trivialization induces a one to one correspondence between $H_{0}^{1}$-Jacobi fields verifying Dirichlet boundary conditions at 0 and $\lambda$ and the weak solutions $f=\left(f_{1}, \ldots, f_{n}\right)$ of the boundary value problem for a second order system of ordinary differential equations

$$
\left\{\begin{array}{l}
J f^{\prime \prime}(x)+S_{\lambda}(x) f(x)=0 \\
f(0)=0=f(1)
\end{array} \quad \forall x \in[0,1]\right.
$$

where $J$ is the symmetry

$$
J=\left(\begin{array}{cc}
\mathrm{I} d_{n-\nu} & 0 \\
0 & -\mathrm{I} d_{\nu}
\end{array}\right)
$$

and $S_{\lambda}(x)=\lambda^{2} S(\lambda x)$, with $S(x)=\left(S_{i j}(x)\right)$ the $n \times n$ symmetric matrix defined by

$$
S_{i j}(x)=g\left(R\left(\gamma^{\prime}(x), e^{i}(x)\right) \gamma^{\prime}(x)+D_{e^{i}(x)} \nabla V(x, \gamma(x)), e^{j}(x)\right) .
$$

Thus weak solutions of the system above are elements in the kernel of the quadratic form $h_{\lambda}: H_{0}^{1}\left([0,1] ; \mathbb{R}^{n}\right) \rightarrow \mathbb{R}$ defined by

$$
h_{\lambda}(f)=\int_{0}^{1}\left\langle J f^{\prime}(x), f^{\prime}(x)\right\rangle-\left\langle S_{\lambda} f(x), f(x)\right\rangle \mathrm{d} x
$$

where $\langle\cdot, \cdot\rangle$ denotes the usual scalar product in $\mathbb{R}^{n}$. In particular $h_{\lambda}$ is a weakly continuous perturbation of a non degenerate form. The quadratic form $h_{\lambda}$ is nothing but the expression of $h_{\gamma_{\lambda}}$ in the trivialization of $\gamma_{\lambda}^{*}[T F(\pi)]$ induced by the trivialization of $\gamma_{\lambda}^{*}(T M)$ given by the parallel frame introduced above (see [35], (3.6)). It follows from this that

$$
\mu(\gamma)=-\operatorname{sf}(h,[0,1]) .
$$

Now let us compute $\operatorname{sf}(h,[0,1])$ assuming that $S(x) \equiv S$ is a constant matrix. We represent, the associated path of bilinear forms against the scalar product $\langle f, g\rangle_{H_{0}^{1}}=\int_{0}^{1}\left\langle f^{\prime}(x), g^{\prime}(x)\right\rangle \mathrm{d} x$ by a path of self-adjoint operators $L$. 
Then, each $L_{\lambda}$ is defined by

$$
\left\langle L_{\lambda} f, g\right\rangle_{H_{0}^{1}}=\int_{0}^{1}\left\langle J f^{\prime}(x) g^{\prime}(x)\right\rangle-\left\langle\lambda^{2} S f(x), g(x)\right\rangle \mathrm{d} x .
$$

Thus, $L_{\lambda}=J+\lambda^{2} K$ where, by abuse of notation, we denote with $J$ the corresponding multiplication operator, while $K$ is the compact operator defined by

$$
\langle K f, g\rangle_{H_{0}^{1}}=-\int_{0}^{1}\langle S f(x), g(x)\rangle \mathrm{d} x .
$$

Any $f \in H_{0}^{1}\left([0,1] ; \mathbb{R}^{n}\right)$ has a Fourier expansion given by

$$
f(x)=\sum_{k=1}^{\infty} a_{k} \sin \pi k x
$$

where $a_{k}$ belong to $\mathbb{R}^{n}$. Hence, $H_{0}^{1}\left([0,1] ; \mathbb{R}^{n}\right)$ decomposes into an orthogonal direct sum

$$
H_{0}^{1}\left([0,1] ; \mathbb{R}^{n}\right)=\bigoplus_{k=1}^{\infty} V_{k}
$$

where

From the relation

$$
V_{k}=\operatorname{span}\left\{a \sin k \pi x: a \in \mathbb{R}^{n}\right\}
$$

$$
K f=g \Longleftrightarrow\left\{\begin{array}{l}
g^{\prime \prime}(x)=S f(x) \\
g(0)=g(1)=0
\end{array}\right.
$$

it follows that each $V_{k}$ is an invariant subspace of $L_{\lambda}$. Since $L_{\lambda}$ is self adjoint $V_{k}$ reduces $L_{\lambda}$ and we have an orthogonal decomposition $L_{\lambda}=\bigoplus_{k=1}^{\infty} L_{\lambda}^{k}$, where $L_{\lambda}^{k}$ is the restriction of $L_{\lambda}$ to $V_{k}$.

If we choose as basis of $V_{k}$ the set $B_{k}$ given by

$$
B_{k}=\left\{\phi_{i, k}(x): 1 \leq i \leq n\right\}, \quad \phi_{i, k}(x)=e_{i} \sin k \pi x,
$$

where $e_{i}$ is the canonical base of $\mathbb{R}^{n}$, then the $n \times n$ matrix that represents $L_{\lambda}^{k}$ with respect to the base $B_{k}$, is given by

$$
L_{\lambda}^{k}=J-\frac{\lambda^{2}}{(\pi k)^{2}} S .
$$

By the above formula the restriction of $L_{\lambda}$ to $\left(\bigoplus_{k=1}^{m} V_{k}\right)^{\perp}$ is an isomorphism for $m$ big enough. Thus by the normalization property (see $[19,35])$, the spectral flow of the restriction of the path $L$ to $\left(\bigoplus_{k=1}^{m} V_{k}\right)^{\perp}$ is zero. Hence,

$$
\operatorname{sf}(L)=\sum_{k=1}^{m} \operatorname{sf}\left(L^{k},[0,1]\right)=\sum_{k=1}^{m}\left[\nu-\mu_{\text {Morse }}\left(L_{1}^{k}\right)\right],
$$

since the spectral flow of a path of operators defined on a finite-dimensional space is the difference between the Morse indices of its end points.

Given a constant symmetric matrix $S$ such that (5.1) has only the trivial solution for $\lambda=1$ we define the index of $S$ with respect to $J$ by

$$
\operatorname{ind}_{J}(S)=\sum_{k=1}^{\infty}\left[\mu_{\text {Morse }}\left(L_{1}^{k}\right)-\nu\right]
$$


Notice that $\operatorname{ind}_{\mathrm{J}}(S)$ is well defined since by the above discussion, the sum on the right hand side is finite.

Summing up the above discussion, we have proved:

Proposition 5.1. If the matrix $S$ defined by (5.3) is constant then $\mu(\gamma)=\operatorname{ind}_{\mathrm{J}}(S)$.

There is a special case, in which the computation of the index turns out to be as simple as in the case of a geodesic on a Riemannian symmetric space.

A matrix $S$ commuting with $J$ will be called split. Any split matrix $S$ has the form

$$
S=\left(\begin{array}{cc}
A & 0 \\
0 & B
\end{array}\right)
$$

where $A$ and $B$ are two symmetric matrices of dimension respectively $(n-\nu) \times(n-\nu)$ and $\nu \times \nu$. Diagonalizing $A$ and $B$ and we get

$$
U^{*} A U=\operatorname{diag}\left(\lambda_{1}, \ldots, \lambda_{n-\nu}\right) \quad \text { and } \quad V^{*} B V=\operatorname{diag}\left(\mu_{1}, \ldots, \mu_{\nu}\right)
$$

and using the invariance of the spectral flow under cogredience we reduce the calculation of ind $\mathrm{J}_{\mathrm{J}}(S)$ to that of the diagonalized system

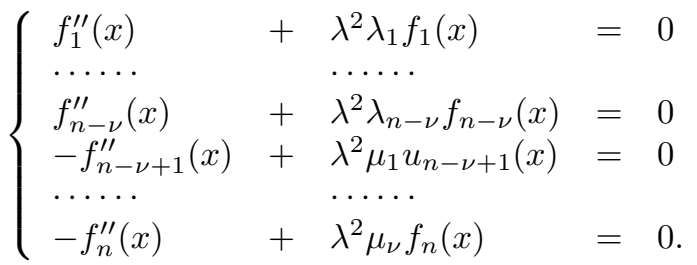

Non trivial solutions for $u^{\prime \prime}+\lambda^{2} a u=0$ with Dirichlet boundary conditions $u(0)=0=u(1)$ arise with multiplicity 1 only for $\lambda^{2} \lambda_{i}=k^{2} \pi^{2}, k \in \mathbb{N}$.

Defining, for a real number $a$,

$$
N(a)=\#\left\{i \in \mathbb{N} \mid i^{2} \pi^{2}<a\right\}
$$

we get:

Proposition 5.2. If $S$ is constant and split then

$$
\mu(\gamma)=\operatorname{ind}_{J}(S)=\sum_{1}^{n-\nu} N\left(\lambda_{i}\right)-\sum_{1}^{\nu} N\left(-\mu_{i}\right)
$$

In general $\operatorname{ind}_{\mathrm{J}}(S)$ can be estimated in terms of the negative real eigenvalues of the $J$-symmetric matrix $J S$. For this we will need some well known results about Maslov index. Most of what we will use here can be found in $[33,44]$.

The Dirichlet boundary value problem for $J u^{\prime \prime}+S u=0$ is equivalent to the following boundary value problem for a first order Hamiltonian system in $\mathbb{R}^{2 n}$

$$
\left\{\begin{array}{l}
w^{\prime}(x)=H w(x) \\
w(0) \in V, w(1) \in V
\end{array}\right.
$$

where $w=(u, v) \in \mathbb{R}^{n} \times \mathbb{R}^{n}, V=\{0\} \times \mathbb{R}^{n}$ and

$$
H=\left(\begin{array}{cc}
0 & J \\
-S & 0
\end{array}\right)
$$

Since $H$ is a hamiltonian matrix its flow $\Psi(x)=e^{x H}$ is a path in the symplectic group $S p(2 n)$ of all transformations preserving the canonical symplectic form $\omega$ on $\mathbb{R}^{2 n}$. The symplectic group acts naturally on the 
manifold $\Lambda(n)$ of all Lagrangian subspaces of $\mathbb{R}^{2 n}$, and hence the path $\Psi$ induces a path $L$ on the Lagrangian Grasmannian $\Lambda(n)$ defined by $L(x)=\Psi(x) V$.

According to [35], Proposition 6.1, if $L(1)$ is transverse to $V$, then $\operatorname{ind}_{\mathrm{J}}(S)$ is defined and, taking $\varepsilon>0$ small enough,

$$
\operatorname{ind}_{\mathrm{J}} S=\mu_{V}(L,[\varepsilon, 1])
$$

where $\mu_{V}(L)$ is the Maslov index of the path $L$ with respect to $V$, an integer which counts algebraically the intersections of the path $L$ with the singular cycle $\Sigma(V)=\left\{V^{\prime} \in \Lambda(n) \mid \operatorname{dim} V \cap V^{\prime}>0\right\}$ or, what is the same, the number of points $x \in[\varepsilon, 1]$ such that $L(x)$ fails to be transverse to $V$. On the other hand, the Maslov index $\mu_{V}(L,[\varepsilon, 1])$ is related with the Conley Zehnder index or the rotation number of the path $\left.\Psi[12,23]\right)$ which can be computed from the spectrum of $J S$. For this, we need to consider the symplectic space $\mathbb{R}^{2 n} \times \mathbb{R}^{2 n}$ equipped with the symplectic form $\bar{\omega}=-\omega \times \omega$. With this symplectic form the path defined by $M(x)=\operatorname{Graph} \Psi(x)$ is a path of Lagrangian subspaces. By [44], Theorem 3.2, if $W=V \times V$ then

$$
\mu_{V}(L,[\varepsilon, 1])=\mu_{W}(M,[\varepsilon, 1])
$$

The Maslov index of the path $M$ with respect to the diagonal $\Delta \subset \mathbb{R}^{2 n} \times \mathbb{R}^{2 n}$ is related to $\mu_{W}(M,[\varepsilon, 1])$ by

$$
\mu_{\Delta}(M,[\varepsilon, 1])=\mu_{W}(M,[\varepsilon, 1])+1 / 2[\operatorname{sign}(W, \Delta, M(1))-\operatorname{sign}(W, \Delta, M(\varepsilon))]
$$

where sign denotes the signature of a triple of Lagrangian subspaces [33].

By our hypothesis $\mathrm{M}(1)$ is transverse to $W$. In what follows, for simplicity, we will assume also that $M(1)$ is transverse to $\Delta$, equivalently, that 1 is not an eigenvalue of $e^{H}$. Notice that both conditions are also verified by $M(\varepsilon)$, if we take $\varepsilon>0$ small enough. Indeed, by [35], Proposition $6.1 M(x)$ fails to be transverse to $W$ only at a conjugate instant and we showed in [35] that conjugate instants cannot accumulate to 0. On the other hand, 1 is an eigenvalue of $e^{\varepsilon H}$ if and only if $2 \frac{\pi}{\varepsilon} i$ is an eigenvalue of $H$ which is impossible for $\varepsilon$ small enough.

In this situation, by [33], Proposition 1,

$$
|\operatorname{sign}(W, \Delta, M(1))| \leq 1 / 2 \operatorname{dim}\left[(\Delta \cap W)^{\omega} / \Delta \cap W\right]
$$

where $(\Delta \cap W)^{\omega}$ denotes the symplectic orthogonal of $\Delta \cap W$. Since $\Delta \cap W$ is an $n$-dimensional isotropic subspace, $|\operatorname{sign}(W, \Delta, M(1))| \leq n$. By the same $\operatorname{argument}|\operatorname{sign}(W, \Delta, M(\varepsilon))| \leq n$. Summing up (5.14), (5.15), (5.16) we obtained

$$
\left|\mu_{\Delta}(M,[\varepsilon, 1])-\operatorname{ind}_{\mathrm{J}} S\right| \leq n .
$$

Let us recall that the Conley-Zehnder index of the path $\Psi:[0, t] \rightarrow S p(n)$ is the integer $\mu_{\Delta}(M,[0, t])$. It depends only on the purely imaginary eigenvalues $H$ and can be computed as follows:

Assume first that all purely imaginary eigenvalues of $H$ are simple. If $\lambda=i \alpha$ is an eigenvalue with eigenvector $e$ then $\bar{e}$ is an eigenvector corresponding to $\bar{\lambda}$. It is easy to see that $\frac{1}{2 i} \omega(\bar{e}, e)$ is a non vanishing real number. Let $\beta= \pm \alpha$ according to the sign of $\frac{1}{2 i} \omega(\bar{e}, e)$. If $\tau=2 \pi / t$ then there exists an $m \in \mathbb{Z}$ such that $m \tau<\beta<(m+1) \tau$. Set $\|\lambda\|=m+1 / 2$ and let $j(t, H)=\sum_{\lambda}\|\lambda\|$ then, by [12],

$$
\mu_{\Delta}(M,[0, t])=j(t, H)
$$

When the imaginary eigenvalues are not simple the above formula still holds provided that we count the imaginary part of each eigenvalue $\lambda$ positively $p$ times and negatively $q$ times according to the number $p$ (resp. q) of positive (resp. negative) eigenvalues of the hermitian form $\frac{1}{2 i} \omega(\bar{e}, e)$ defined on the generalized eigenspace of $\lambda$.

With this we can estimate $\operatorname{ind}_{\mathrm{J}}(S)$ in terms of the negative eigenvalues of the matrix $J S$. Indeed, since $H^{2}=\operatorname{diag}(J S, S J)$ the purely imaginary eigenvalues of $H$ are precisely square roots of the negative eigenvalues of $J S$. Let $j(S)=j(1, H)-j(\varepsilon, H)$. 
From (5.18) and the above computation we get $\left|\operatorname{ind}_{\mathrm{J}}(S)-j(S)\right| \leq n$ and therefore we obtain:

Proposition 5.3. Under the above assumptions

$$
j(S) / n-1 \leq \operatorname{ind}_{\mathrm{J}}(S) / n \leq j(S) / n+1 .
$$

Since our main theorem allows us to estimate the number of bifurcation points in terms of $\operatorname{ind}_{\mathrm{J}}(S) / n$, the above result is good enough for our purpose.

\section{Applications}

A semi Riemannian manifold $(M, g)$ is locally symmetric if for any $p \in M$, there is a local isometry $s_{p}$ at $p$, leaving $p$ fixed and such that $T_{p} s_{p}=-\mathrm{Id}$. The above condition is equivalent to the vanishing of the covariant derivative of the curvature tensor or, what is the same, that the curvature tensor commutes with parallel translation along paths on $M$.

The curvature operator along a path $\gamma$ is the $g$-symmetric endomorphism $R_{\gamma}(x): T_{\gamma(x)} M \rightarrow T_{\gamma(x)} M$, defined by

$$
R_{\gamma}(x)(v)=R\left(\gamma^{\prime}(x), v\right) \gamma^{\prime}(x)
$$

Notice that here we follow Klingerberg's book [29] in the choice of terminology. Elsewhere this operator is called either "tidal force" or "Jacobi operator" while the term "curvature operator" is reserved to the curvature tensor viewed as a function of the third variable, i.e. to the infinitesimal generators of the restricted holonomy.

By the above discussion the curvature operator along any geodesic path $\gamma$ on a locally symmetric manifold $M$ commutes with the parallel translation along $\gamma$ and therefore the matrix

$$
\left(R_{i, j}(x)\right)=g\left(R\left(\gamma^{\prime}(x), e^{i}(x)\right) \gamma^{\prime}(x), e_{j}(x)\right)
$$

of $R_{\gamma^{\prime}(x)}$ in any parallel orthonormal frame $\mathcal{F}=\left\{e^{i}(x) ; i=1, \ldots, n\right\}$ is constant. The index of the matrix $\left(R_{i, j}\right)$ is clearly independent from the choice of the frame and will be denoted by $\operatorname{ind}_{\mathrm{J}} R$.

From Theorem 4.2 and Proposition 5.1 (with $V \equiv 0$ ) we obtain:

Corollary 6.1. Let $(M, g)$ be an analytic semi Riemannian locally symmetric space. If $\gamma$ is a non degenerate geodesic there are at least $\left|\operatorname{ind}_{\mathrm{J}}(R)\right| /(n-1)$ strictly conjugate points along $\gamma$.

Let $(M, g)=\left(M_{1}, g_{1}\right) \times\left(M_{2},-g_{2}\right)$ where $M_{i}$ are Riemannian symmetric spaces of dimension $n-\nu$ and $\nu$ respectively. If $\gamma$ is a non degenerate geodesic on $M$, the curvature operator along $\gamma$ splits into a direct sum of the curvature operators along projections of $\gamma$ on $M_{1}$ and $M_{2}$. Denoting the corresponding eigenvalues by $\lambda_{i}$ and $\mu_{i}$ respectively, from Proposition 5.2 we get

Corollary 6.2. If $M$ and $\gamma$ are as above, then along $\gamma$ there are at least

$$
\left|\sum_{i=1}^{n-\nu} N\left(\lambda_{i}\right)-\sum_{i=1}^{\nu} N\left(-\mu_{i}\right)\right| /(n-1)
$$

strictly conjugate points.

Now we consider bifurcation of $p$-geodesics of a family of analytic mechanical systems $\left(M, g_{\lambda}, V_{\lambda}\right)$ parametrized by $I=[a, b]$.

Assume that both $V_{a}$ and $V_{b}$ are time independent. Let $q_{a}$ and $q_{b}$ be critical points of $V_{a}$ and $V_{b}$ respectively. The constant paths $q_{a}$ and $q_{b}$ are $p$-geodesics for the corresponding mechanical systems. Let $H_{i}(v)=D_{v} \nabla V_{i}\left(q_{i}\right): T_{q_{i}} M \rightarrow T_{q_{i}} M ; i=a, b$ be the Hessians of $V_{i}$ at $q_{i}$. Since the curvature operator along a constant path vanishes, the matrix $S$ of (5.3) reduces in this case to the matrix of the Hessian in an orthonormal basis of $T_{q_{i}} M$. From the main theorem we obtain: 
Corollary 6.3. Let $\left(M, g_{\lambda}, V_{\lambda}\right)$ be as above. Assume that the constant p-geodesics $q_{a}$ and $q_{b}$ are non degenerate. Then, along any analytic trivial branch $\sigma$ of $p$-geodesics such that $\sigma_{a}=q_{a}$ and $\sigma_{b}=q_{b}$ there must be at least $\left|\operatorname{ind}_{\mathrm{J}}\left(H_{a}\right)-\operatorname{ind}_{\mathrm{J}}\left(H_{b}\right)\right| / n$ bifurcation points.

Our next result deals with bifurcation of nontrivial periodic $p$-geodesics from a branch of critical points on symmetric spaces.

Let $\left(M, g_{\lambda}, V_{\lambda}\right)$ be as above. Assume moreover that $M$ is an analytic semi Riemannian symmetric space. Suppose also that each $V_{\lambda}$ is time independent and that the trivial branch of $p$-geodesics is given by an analytic path $q: I \rightarrow M$ such that each $q_{\lambda}$ is a critical point of $V_{\lambda}$. Assume that the potential $V_{\lambda}$ is invariant under the symmetry $s_{\lambda}$ at $q_{\lambda}$.

Corollary 6.4. Under the above assumptions, if $q_{a}$ and $q_{b}$ are non degenerate $p$-geodesics then there are at least $\left|\operatorname{ind}_{\mathrm{J}}\left(H_{a}\right)-\operatorname{ind}_{\mathrm{J}}\left(H_{b}\right)\right| / n$ points of the branch at which bifurcation of non constant 2-periodic geodesics arises.

By the above corollary, there are at least $\left|\operatorname{ind}_{J}\left(H_{a}\right)-\operatorname{ind}_{J}\left(H_{b}\right)\right| / n$ points $\lambda_{*} \in I$ such that close to the constant $p$-geodesic $q_{\lambda^{*}}$ there are $p$-geodesics with both end points on the trivial branch but not belonging to the trivial branch. Such geodesics cannot be constant.

On the other hand, since $s_{\lambda}$ is an isometry and $V_{\lambda} s_{\lambda}=V_{\lambda}$, the energy functional $E_{\lambda}$ is invariant under the map induced on $\Omega_{\lambda}$ by $s_{\lambda}$. Hence, if $\gamma$ is any $p$-geodesic for $\left(g_{\lambda}, V_{\lambda}\right)$, so is $s_{\lambda} \gamma$. By symmetry any non constant $p$-geodesic $\gamma$ with end points at $q_{\lambda}$ and close to the trivial branch produces a non constant periodic geodesic $\bar{\gamma}$ of period 2 close to the branch $q$. For this, it is enough to define

$$
\bar{\gamma}(x)=\left\{\begin{array}{ccc}
\gamma(x) & \text { if } \quad 0 \leq x \leq 1 \\
s_{\lambda} \gamma(2-x) & \text { if } \quad 1 \leq x \leq 2
\end{array}\right.
$$

Observe that Corollary 6.4 it is not applicable to geodesics, since it holds true only if the potential is not trivial.

\section{A COMPARISON PRINCIPLE FOR $p$-GEODESICS ON SEMI-RIEMANNIAN MANIFOLDS}

Given a mechanical system $(M, g, V)$ and a path $\gamma:[0,1] \rightarrow M$ let us consider

$$
S_{\gamma}(x): T_{\gamma(x)} M \rightarrow T_{\gamma(x)} M ; S_{\gamma}(x)=R_{\gamma}(x)+P_{\gamma}(x)
$$

where $R_{\gamma}(x)(v)=R\left(\gamma^{\prime}(x), v\right) \gamma^{\prime}(x)$ and $P_{\gamma}(x)(v)=D_{v} \nabla V(x, \gamma(x))$ are the curvature operator and the Hessian of $V$ along $\gamma$ respectively.

Since both $R_{\gamma}$ and $P_{\gamma}$ are $g$-symmetric it follows that $S_{\gamma}$ is a symmetric endomorphism of $\gamma^{*}(T M)$.

Let $\left(M_{1}, g_{1}, V_{1}\right)$ and $\left(M_{2}, g_{2}, V_{2}\right)$ be two mechanical systems on manifolds of the same dimension $n$ and such that both $g_{1}$ and $g_{2}$ have the same index $\nu$. Let $p_{i} \in M_{i} ; i=1,2$ be two given points. Choose an isometry $\varphi: T_{p_{1}} M_{1} \rightarrow T_{p_{2}} M_{2}$. Given $v_{1} \in T_{p_{1}} M_{1}$, let $\gamma_{1}$ be the unique $p$-geodesic corresponding to $\left(g_{1}, V_{1}\right)$, such that $\gamma_{1}(0)=p_{1}$ and $\gamma_{1}^{\prime}(0)=v_{1}$. If $v_{2}=\varphi\left(v_{1}\right)$, we denote by $\gamma_{2}$ the unique $p$-geodesic in $M_{2}$ for the system $\left(g_{2}, V_{2}\right)$, such that $\gamma_{2}(0)=p_{2}$ and $\gamma_{2}^{\prime}(0)=v_{2}$. We assume that both $\gamma_{1}$ and $\gamma_{2}$ are defined on the interval [0,1] and are nondegenerate critical points of the corresponding energy functionals.

We will compare the index of $\gamma_{1}$ with the index of $\gamma_{2}$. First we extend the isometry $\varphi$ to an isometry between $\gamma_{1}^{*}(T M)$ and $\gamma_{2}^{*}(T M)$. For any $x \in[0,1]$, let $\varphi_{x}: T_{\gamma_{1}(x)} M_{1} \rightarrow T_{\gamma_{2}(x)} M_{2}$ be defined by

$$
\varphi_{x} v=\mathcal{P}_{x}^{2} \circ \varphi \circ \mathcal{P}_{-x}^{1}(v) \quad \forall v \in T_{\gamma_{1}(x)} M_{1}
$$

where $\mathcal{P}_{x}^{i}: T_{p_{i}} M_{i} \rightarrow T_{\gamma_{i}(x)} M_{i}$ is the parallel transport along $\gamma_{i}$. 
Theorem 7.1. Let $\left(M_{i}, g_{i}, V_{i}\right)$ and $\gamma_{i}$ be as above. Let $S^{i}(x)=S_{\gamma_{i}}(x)$. If for each $x \in[0,1]$ and $v \in T_{\gamma_{1}(x)}$

$$
g_{2}\left(S^{2}(x) \varphi_{x} v, \varphi_{x} v\right) \leq g_{1}\left(S^{1}(x) v, v\right)
$$

then

$$
\mu\left(\gamma_{2}\right) \leq \mu\left(\gamma_{1}\right)
$$

When both manifolds are Riemannian, the Hessian forms are essentially positive and have a finite Morse index. In this case the theorem is an easy consequence of the fact that Morse index is nonincreasing. The proof in the general case is based on monotonicity properties of the spectral flow and it is given in Section 9. Analogous results can be found in [3] and [4] in a different framework.

Taking $V \equiv 0$ we obtain an extension of the Morse-Schöenberg comparison theorem to general semi Riemannian manifolds.

Corollary 7.2. Let $\left(M_{i}, g_{i}\right)$ and $\gamma_{i}$ be as above with $V_{i} \equiv 0$. If for each $x \in[0,1]$,

$$
g_{2}\left(R_{\gamma_{2}}(x) \varphi_{x} v, \varphi_{x} v\right) \leq g_{1}\left(R_{\gamma_{1}}(x) v, v\right)
$$

then $\mu\left(\gamma_{2}\right) \leq \mu\left(\gamma_{1}\right)$.

For lightlike and timelike geodesics on Lorentzian manifolds this was proved by Beem Ehrlich Easley (see for instance [5]).

Comparing an analytic semi Riemannian manifold $M_{1}$ with a semi Riemannian locally symmetric space $M_{2}$ of the same dimension and index we conclude that.

Corollary 7.3. If (7.4) holds and if $\operatorname{ind}_{\mathrm{J}} R_{2}(0)$ is defined and is positive, then along $\gamma_{1}$ there must be at least $\operatorname{ind}_{\mathrm{J}} R_{2}(0) /(n-1)$ strictly conjugate points. The same is true if $\operatorname{ind}_{\mathrm{J}} R_{2}(0)$ is negative and the reverse of inequality (7.4) holds.

The simplest comparison is with a space form $M_{2}$ of index $\nu$ and curvature $K$. The curvature tensor of $M_{2}$ is $R_{2}(X, Y) Z=K\left[g_{2}(Z, X) Y-g_{2}(Z, Y) X\right]$. Since $\varphi_{x}$ is an isometry sending $\gamma_{1}^{\prime}(x)$ into $\gamma_{2}^{\prime}(x)$ we have

$$
g_{2}\left(R_{\gamma_{2}}(x) \varphi_{x} v, \varphi_{x} v\right)=K\left[g_{1}\left(\gamma_{1}^{\prime}(x), \gamma_{1}^{\prime}(x)\right) g_{1}(v, v)-g_{1}\left(\gamma_{1}^{\prime}(x), v\right)^{2}\right]
$$

Identifying $T_{p_{1}} M_{1}$ with $T_{p_{2}} M_{2}$ via $\varphi$, the curvature operator of $R_{2}$ restricted to the normal subspace $\gamma_{2}^{\prime}(0)^{\perp}$ coincides with $K\left[g_{1}\left(\gamma_{1}^{\prime}(0), \gamma_{1}^{\prime}(0)\right)\right]$ Id on $\gamma_{1}^{\prime}(0)^{\perp}$. If $\gamma_{1}$ is not a null geodesic, without loss of generality we can assume that $\epsilon\left(\gamma_{1}\right)=g_{1}\left(\gamma_{1}^{\prime}(0), \gamma_{1}^{\prime}(0)\right)= \pm 1$. By Proposition $5.2 \operatorname{ind}_{\mathrm{J}} R_{2}=N\left(K \epsilon\left(\gamma_{1}\right)\right)(n-1)$. From the above corollary we conclude that

Corollary 7.4. If $(M, g)$ is a semi Riemannian manifold such that

$$
g(R(X, Y) Y, X) \geq K\left[g(X, X) g(Y, Y)-g(X, Y)^{2}\right]
$$

for some real number $K$, then any geodesic $\gamma$ on $M$ such that $K \epsilon(\gamma)>0$ has at least $N(K \epsilon(\gamma))$ strictly conjugate points.

If the manifold is Riemannian, inequality (7.5) implies that the sectional curvature has to be bounded from below. When the metric is indefinite, if the sectional curvature is bounded from below (or above) then it is automatically bounded. Nevertheless, the hypothesis in Corollary 7.4 does not imply that the sectional curvature of $M$ is constant. Nontrivial examples of semi Riemannian manifolds verifying (7.5) are given in [2]. In this paper and also in [31] several comparison results of Sturm type are proved.

In a similar way we can obtain estimates for the number of strictly conjugate points along a nondegenerate horizontal geodesic in a semi Riemannian warped product with locally symmetric base. 
Let $B$ and $F$ be two semi Riemannian manifolds and let $\rho$ be a smooth positive function defined on $B$. The warped product $M_{\rho}=B \times{ }_{\rho} F$ is the product manifold $B \times F$ endowed with a twisted product metric. Namely, for $p=(b, f) \in B \times F$ we write the tangent space $T_{p} B \times F$ as an internal direct sum of $T_{p} B \equiv T_{(b, f)} B \times\{f\}$ with $T_{p} F \equiv T_{(b, f)}\{b\} \times F$ and endow it with the metric $g_{\rho}=g_{B}+\rho^{2} g_{F}$. Notice that the direct sum decomposition $T_{p} M_{\rho}=T_{p} B \oplus T_{p} F$ is orthogonal with respect to both $g_{\rho}$ and the product metric $g_{1}$.

We will do the comparison between the manifold $M_{\rho}$ and the manifold $M_{1}$, the semi Riemannian product of $B$ and $F$. Given a point $p=(b, f)$ we choose an isometry $\varphi: T_{p} M_{\rho} \rightarrow T_{\varphi(p)} M_{1}$ whose restriction to $T_{p} B$ is the identity.

Let $\gamma$ be any horizontal geodesic through $p$. Then $\gamma$ is a geodesic under both metrics and decomposing a vector $w \in T_{\gamma(x)}$ as $w=(u, v)$ under the above orthogonal decomposition we have that the curvature operator on $M_{1}$ is given by

$$
R_{\gamma}(x)(u, v)=R\left(\gamma^{\prime}(x), u\right) \gamma^{\prime}(x)=R_{\bar{\gamma}}^{B}(x) u
$$

where $\bar{\gamma}$ is the projection of $\gamma$ onto the base $B$ and $R^{B}$ is the curvature operator on $B$.

It follows plainly from (7.6) that the family of hessians $h_{\lambda}$ of the energy functional on $M_{1}$ at $\gamma_{\lambda}, \lambda \in[0,1]$ is a direct sum of the family of hessians $\bar{h}_{\lambda}$ of $E$ on $B$ at $\bar{\gamma}_{\lambda}$ with a family of nondegenerate quadratic forms defined on $T_{\gamma_{\lambda}}(F)$. Hence, by normalization and the direct sum property of the spectral flow, $\mu(\gamma)=\bar{\mu}(\bar{\gamma})$, as a geodesic on $M_{1}$.

On the other hand by [36], Proposition 42 , on $M_{\rho}$ we have

$$
R_{\gamma}(x)(u, v)=R\left(\gamma^{\prime}(x), u\right) \gamma^{\prime}(x)-\frac{1}{f} \operatorname{Hess}(\rho)\left(\gamma^{\prime}(x), \gamma^{\prime}(x)\right) v
$$

From Corollary 7.2 and the above discussion it follows that if $\rho$ is a convex function then for any horizontal geodesic $\gamma$ on $M_{\rho}$ we have $\mu(\bar{\gamma}) \leq \mu(\gamma)$. Hence:

Corollary 7.5. If $B$ is a locally symmetric space and the warping function $\rho$ is convex then along any nondegenerate horizontal geodesic there are at least $\operatorname{ind}_{\mathrm{J}} R_{\bar{\gamma}}^{B}(0) /(n-1)$ strictly conjugate points.

Our final application is an estimate of the number of bifurcation points from the trivial branch for a family $\left(M, g, V_{\lambda}, \sigma_{\lambda}\right)$ where $\mathrm{M}$ is an analytic complete locally symmetric space and $V_{\lambda}, \lambda \in[a, b]$, is an analytic family of potentials on $M$. For $i=a, b$ let $R_{i}(x): T_{\sigma_{i}(x)} M \rightarrow T_{\sigma_{i}(x)} M$ and $\mathcal{P}_{x}^{i}: T_{\sigma_{i}(0)} M \rightarrow T_{\sigma_{i}(x)} M$ be the curvature operator and the parallel translation along the $p$-geodesic $\sigma_{i}$ respectively.

Theorem 7.6. Assume that, as symmetric operators,

$$
\mathcal{P}_{x}^{a} R_{a}(0) \mathcal{P}_{-x}^{a} \geq R_{a}(x) a n d \mathcal{P}_{x} R_{b}(0) \mathcal{P}_{-x} \leq R_{b}(x)
$$

Suppose moreover that the hessians of $V_{i}, i=a, b$ at each point of $\sigma_{i}$ are respectively positive and negative semidefinite. Then, if $\operatorname{ind}_{\mathrm{J}}\left(R_{a}\right)(0)>\operatorname{ind}_{\mathrm{J}}\left(R_{b}\right)(0)$, the number of bifurcation points from the trivial branch along $\sigma$ is not less than $\left.\operatorname{ind}_{\mathrm{J}}\left(R_{a}(0)\right)-\operatorname{ind}_{\mathrm{J}}\left(R_{b}(0)\right)\right] / n$.

Proof. We use the comparison principle between $\left(M, g, V_{i}\right)$ and $(M, g, 0)$. Comparing the Hessian of $E$ at the $p$-geodesics $\sigma_{i}$ with the Hessian of the energy at a geodesic having the same origin and initial velocity, we obtain

$$
\mu\left(\sigma_{a}\right) \geq \operatorname{ind}_{\mathrm{J}}\left(R_{a}\right)(0) \text { and } \mu\left(\sigma_{b}\right) \leq \operatorname{ind}_{\mathrm{J}}\left(R_{b}\right)(0)
$$

Therefore

$$
\left|\mu\left(\sigma_{a}\right)-\mu\left(\sigma_{b}\right)\right| \geq \operatorname{ind}_{\mathrm{J}}\left(R_{a}\right)(0)-\operatorname{ind}_{\mathrm{J}}\left(R_{b}\right)(0) .
$$

The conclusion follows from the main theorem. 


\section{Proof of the MAin theOrem}

Before going to the proof of Theorem 4.2 we will prove an abstract bifurcation theorem for critical points of families of smooth functionals. This theorem is an extension of the main theorem in [19] to the geometric framework which naturally arises in dealing with bifurcation of $p$-geodesics. Moreover, we will improve the result in [19] obtaining estimates from below for the number of bifurcation points.

Definition 8.1. A smooth family of Hilbert manifolds $\left\{X_{\lambda}\right\}_{a \leq \lambda \leq b}$ parameterized by a real interval $I=[a, b]$ is a family of manifolds of the form $X_{\lambda}=p^{-1}(\lambda)$ where $p: X \rightarrow \bar{I}$ is a smooth submersion of a Hilbert manifold $X$ onto $I$.

By the implicit function theorem each fiber $X_{\lambda}$ of the submersion is a submanifold of $X$ of codimension one. For each $x \in X_{\lambda}$ the tangent space $T_{x} X_{\lambda}$ coincides with $\operatorname{ker} T p_{x}$. Being $p$ a submersion the family of Hilbert spaces $T F(p)=\left\{\operatorname{ker} T p_{x}: x \in X\right\}$ is a Hilbert sub-bundle of the tangent bundle $T X$. $T F(p)$ is the bundle of tangents along the fibers or the vertical bundle of the submersion $p$. A smooth functional $f: X \rightarrow \mathbb{R}$ defines by restriction to the fibers of $p$ a smooth family of functionals $f_{\lambda}: X_{\lambda} \rightarrow \mathbb{R}$.

We will assume that there exists a smooth section $\sigma: I \rightarrow X$ of $p$ such that $\sigma(\lambda)$ is a critical point of the restriction $f_{\lambda}$ of the functional $f$ to the fiber $X_{\lambda}$.

We will refer to $\sigma$ as the trivial branch of critical points of the family $\left\{f_{\lambda}: \lambda \in I\right\}$. We will say that $\lambda_{*} \in I$ is a point of bifurcation for critical points of the family $\left\{f_{\lambda}: \lambda \in I\right\}$ from the trivial branch $\sigma(I)$ if there exists a sequence $\lambda_{n} \rightarrow \lambda_{*}$ and a sequence $x_{n} \rightarrow \sigma\left(\lambda_{*}\right)$ such that $p\left(x_{n}\right)=\lambda_{n}$ and each $x_{n}$ is a critical point of $f_{\lambda_{n}}$ not belonging to $\sigma(I)$.

In what follows we will denote with $h_{\lambda}$ the Hessian of $f_{\lambda}$ at the point $\sigma(\lambda)$.

Our next assumption is that, for each $\lambda \in I$, the Hessian $h_{\lambda}$ is a Fredholm quadratic form. Moreover we will assume that the end points $h_{a}$ and $h_{b}$ are non degenerate.

The family of Hessians $h_{\lambda}$, for $\lambda \in[a, b]$, defines a smooth function $h$ on the total space of the pull-back bundle $\mathcal{H}=\sigma^{*} T F(p)$ of the vertical bundle $T F(p)$ by the map $\sigma: I \rightarrow X$ such that the restriction of $h$ to each fiber is a Fredholm quadratic form. The function $h: \mathcal{H} \rightarrow \mathbb{R}$ is a generalized family of Fredholm quadratic forms. Since the restrictions $h_{a}, h_{b}$ to the fibers over $a$ and $b$ are non degenerate, the family is admissible and the spectral flow $\operatorname{sf}(h)=\operatorname{sf}(h,[a, b])$ of the family $h$ is defined by [35], (2.2).

Theorem 8.2. Under the above assumptions if $\operatorname{sf}(h) \neq 0$, then there exists at least one bifurcation point $\lambda_{*} \in(a, b)$ of critical points of $f$ from the trivial branch.

Moreover, if $\operatorname{ker} h_{\lambda} \neq 0$ only for a number of points $\lambda \in I$ then there are at least $|\operatorname{sf}(h)| / m$ distinct bifurcation points in $(a, b)$, where $m=\max \left\{\operatorname{dim} \operatorname{ker} h_{\lambda}\right\}$.

Let us mention that the singular set $\Sigma(h)=\left\{\lambda \in I: \operatorname{ker} h_{\lambda} \neq 0\right\}$ is finite if all the data $(X, p, f, \sigma)$ are analytic. Another situation in which $\Sigma(h)$ is finite is the following one:

If $h: \mathcal{H} \rightarrow \mathbb{R}$ is a generalized family of quadratic forms, the intrinsic derivative $\dot{h}_{\lambda}: \mathcal{H}_{\lambda} \rightarrow \mathbb{R}$ of $h$ at $\lambda$ is the restriction to $\operatorname{ker} h_{\lambda}$ of the ordinary derivative of the smooth path of quadratic forms $h_{\lambda} M_{\lambda}$ where $M$ is any local trivialization of the bundle $\mathcal{H}$. It is easy to see that the resulting quadratic form $\dot{h}_{\lambda}$ is independent from the choice of trivialization. A point $\lambda \in \Sigma(h)$ is called regular when $\dot{h}_{\lambda}$ is a nondegenerate quadratic form on ker $h_{\lambda}$. By straightforward extension to bundles of the arguments in [20], one shows that each regular point $\lambda \in \Sigma(h)$ is isolated in $\Sigma(h)$. Therefore, if $h$ has only regular singular points they are in finite number.

Remark 8.3. By [20] if all points in $\Sigma(h)$ are regular then

$$
\operatorname{sf}(h)=\sum_{\lambda \in \Sigma} \operatorname{sign} \dot{h}_{\lambda}
$$

where sign denotes the signature. 
Proof of Theorem 8.2. By the vector bundle neighborhood theorem (Th. 9) there exist a trivial Hilbert bundle $\mathcal{H}=I \times H$ over $I=[a, b]$ and a fiber preserving smooth map $\psi: \mathcal{H} \rightarrow \sigma^{*} X$ such that $\psi(\lambda, 0)=\sigma(\lambda)$ and such that $\psi$ is a diffeomorphism of $\mathcal{H}$ with an open neighborhood $\mathcal{O}$ of $\sigma(I)$ in $X$.

Let $\tilde{f}: I \times H \rightarrow \mathbb{R}$ be defined by $\tilde{f}=f \circ \psi$. Then $\tilde{f}$ is a family of smooth functionals on the Hilbert space $H$. Since the restriction $\psi_{\lambda}$ of $\psi$ to the fiber is a diffeomorphism, we have that $u \in H$ is a critical point of $\tilde{f}_{\lambda}=f_{\lambda} \circ \psi_{\lambda}$ if and only if $x=\psi_{\lambda}(u)$ is a critical point of $f_{\lambda}$. In particular 0 is a critical point of $\tilde{f}_{\lambda}$ for each $\lambda \in I$. The Hessian $\tilde{h}_{\lambda}$ of $\tilde{f}_{\lambda}$ at 0 is given by $\tilde{h}_{\lambda}(\xi)=h_{\lambda}\left(T_{0} \psi_{\lambda}\right)([\xi])$. By cogredience and normalization property of the spectral flow (see Props. 3.1 and 3.2 in [19]), we have that $\operatorname{sf}(h, I)=\operatorname{sf}(\tilde{h}, I)=\operatorname{sf}(L, I)$ where $L: I \rightarrow \Phi_{s}(H)$ is the smooth path of self-adjoint Fredholm operators representing the family $\tilde{h}$ with respect to the scalar product, i.e. $\tilde{h}_{\lambda}(u)=\left\langle L_{\lambda} u, u\right\rangle \quad \forall u \in H$.

In order to prove the first assertion we argue as follows. Our hypothesis implies that $\operatorname{sf}(L, I) \neq 0$, and [19], Theorem 1, ensures the existence of a critical point $u \neq 0$ of some $f_{\lambda}, \lambda \in I$, in every neighborhood $V$ of 0 . Choosing a $u_{n} \neq 0$ in $V_{n}=B(0,1 / n)$ we can assume by passing, if necessary, to subsequences that the corresponding sequence $\lambda_{n}$ converges to some $\lambda_{*} \in(a, b)$. Let $x_{n}=\psi_{\lambda_{n}}\left(u_{n}\right)$. By construction of $\psi$ each $x_{n}$ is a critical point of $f_{\lambda_{n}}$ not belonging to $\sigma(I)$ and $x_{n} \rightarrow \sigma\left(\lambda_{*}\right)$. Thus $\lambda_{*}$ is a bifurcation point.

In order to prove the second assertion let us assume that the set $\Sigma(h)$ is finite. Let $a<\lambda_{1}<\lambda_{2}<\ldots<\lambda_{k}<b$ be the points in $\Sigma(h)$. We want to show that at least $|\operatorname{sf}(h)| / m$ of them are bifurcation points.

Taking $I_{j}=\left[\lambda_{j}-\delta, \lambda_{j}+\delta\right]$ with $\delta>0$ sufficiently small so that $I_{j}$ are disjoint, we have that $h$ at the end points of $I_{j}$ is non degenerate and hence

$$
\operatorname{sf}(h, I)=\sum_{j=1}^{k} \operatorname{sf}\left(h, I_{j}\right)
$$

by additivity of the spectral flow. Since 0 is always an isolated point in the spectrum of a self-adjoint Fredholm operator, there exists a positive number $\eta$ such that the spectrum of $L_{\lambda_{j}}$ intersects the interval $[-\eta, \eta]$ only in 0 . Then we can choose $\delta>0$ small enough so that $\eta,-\eta$ do not belong to the spectrum of $L_{\lambda}$, for any $\lambda \in I_{j}$ and so that (8.1) still holds true. Let $P_{\lambda}, \lambda \in\left[\lambda_{j}-\delta, \lambda_{j}+\delta\right]$, be the orthogonal projection onto the subspace associated with the part of the spectrum of $L_{\lambda}$ lying in the interval $[-\eta, \eta]$. Taking if necessary a smaller $\delta$, by [27], Chapter II, Section 6 , there exists a continuously differentiable path $U$ on $\left[\lambda_{j}-\delta, \lambda_{j}+\delta\right]$ of orthogonal operators such that

$$
U_{\lambda_{j}}=I d \quad \text { and } \quad U_{\lambda} P_{\lambda} U_{\lambda}^{-1}=P_{\lambda_{j}} \quad \forall \lambda \in\left[\lambda_{j}-\delta, \lambda_{j}+\delta\right] .
$$

Since the spectral flow is invariant under cogredience, we get

$$
\operatorname{sf}\left(L_{\lambda}, I_{j}\right)=\operatorname{sf}\left(M_{\lambda}, I_{j}\right)
$$

where $M_{\lambda}=U_{\lambda} L_{\lambda} U_{\lambda}^{-1}$.

From (8.2), it follows that $M_{\lambda}$ is reduced by $P_{\lambda_{j}}$, for any $\lambda \in I_{j}$, being $L_{\lambda}$ reduced by $P_{\lambda}$ in that interval. Under the splitting $H=\operatorname{ker} L_{\lambda_{j}} \bigoplus \operatorname{Im} L_{\lambda_{j}}, M_{\lambda}$ can be written as $M_{\lambda}=M_{\lambda}^{1}+M_{\lambda}^{2}$. But $M_{\lambda}^{2}$ is an isomorphism and therefore $\operatorname{sf}\left(M_{\lambda}^{2}, I_{j}\right)=0$.

From this and (8.3) we get

$$
\operatorname{sf}\left(L_{\lambda}, I_{j}\right)=\operatorname{sf}\left(M_{\lambda}^{1}, I_{j}\right)=\mu_{\text {Morse }}\left(M_{\lambda_{j}-\delta}^{1}\right)-\mu_{\text {Morse }}\left(M_{\lambda_{j}+\delta}^{1}\right)
$$

where the last equality follows from the property that the spectral flow of a path of linear operators defined on a finite dimensional space equals the difference of the Morse indexes at the end points.

Since $\operatorname{dim} \operatorname{ker} L_{\lambda_{j}} \leq m$ it follows that

$$
\left|\mu_{\text {Morse }}\left(M_{\lambda_{j}-\delta}^{1}\right)-\mu_{\text {Morse }}\left(M_{\lambda_{j}+\delta}^{1}\right)\right| \leq m ;
$$


Summing up

$$
|\operatorname{sf}(h)| \leq \sum_{j=1}^{k}\left|\operatorname{sf}\left(L_{\lambda_{j}}, I_{j}\right)\right| \leq \mathrm{d} m
$$

where $\mathrm{d}$ is the number of nonzero addends in the above formula. Since, by the first part, any interval $I_{j}$ with a nontrivial contribution to the above sum must contain at least one bifurcation point there must be at least $d=|\operatorname{sf}(h)| / m$ distinct bifurcation points in $I$.

With this we can finally prove Theorem 4.2 .

Let us consider the end-point map $e: I \rightarrow M \times M$ defined by $e(\lambda)=(\sigma(\lambda)[0], \sigma(\lambda)[1])$. The pullback $e^{*}(\pi): e^{*}(\Omega) \rightarrow I$ of the submersion $\pi: \Omega \rightarrow M \times M$ via the map $e$ is defined in the usual way. By standard transversality arguments we have that the total space $e^{*}(\Omega)=\{(\lambda, \gamma) \in I \times \Omega: e(\lambda)=\pi(\gamma)\}$ is an Hilbert manifold (which inherits the Riemannian metric from $\Omega$ ) and the map $e^{*}(\pi): e^{*}(\Omega) \rightarrow I$ given by the projection to the first factor is a submersion (see [32], Chap. 3, Sect. 3).

The fiber of $e^{*}(\pi)$ over $\lambda \in I$ is the submanifold $\Omega_{\sigma(\lambda)[0], \sigma(\lambda)[1]}$. By the commutativity of the following diagram

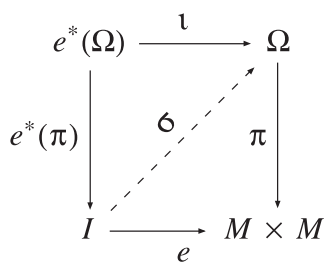

where $\iota$ is the projection to the second factor, we have that $\sigma: I \rightarrow \Omega$ induces a section $\bar{\sigma}: I \rightarrow e^{*}(\Omega)$ of $e^{*}(\pi)$. By the same argument the vertical bundle $T F\left(e^{*}(\pi)\right)$ coincides with $\iota^{*}(T F(\pi))$ and moreover $\sigma^{*}(T F(\pi))=$ $\bar{\sigma}^{*}\left[T F\left(e^{*}(\pi)\right)\right]$. The one-parameter family of functionals $\bar{E}=E \circ \iota: e^{*}(\Omega) \rightarrow \mathbb{R}$ has the same bifurcation points as the family $\left\{E_{\lambda}: \lambda \in I\right\}$ because $\iota_{\lambda}$ sends in a one to one fashion critical points of $\bar{E}_{\lambda}$ into critical points of $E_{\lambda}$. Moreover the isomorphism $\iota_{\lambda}$ intertwines the Hessian $\bar{h}_{\lambda}$ of $\bar{E}_{\lambda}$ at $\bar{\sigma}_{\lambda}$ with the Hessian $h_{\lambda}$ of $E_{\lambda}$ at $\sigma_{\lambda}$. Now Theorem 4.2 follows from Theorem 8.2 and the following proposition

Proposition 8.4. Under assumptions of Theorem 4.2,

$$
\operatorname{sf}(h)=\mu\left(\sigma_{a}\right)-\mu\left(\sigma_{b}\right)
$$

Proof. Let $T=[a, b] \times[0,1]$. Consider the map $\theta: T \rightarrow \Omega$ defined by

$$
\theta(\lambda, t)(x)=\sigma_{\lambda}(t x)
$$

Each $\theta(\lambda, t)$ is a critical point of the energy functional $E_{\lambda, t}: \Omega_{e(\lambda)} \rightarrow \mathbb{R}$ associated to the data $\left(M, g_{\lambda}, t^{2} V_{\lambda}\right)$. Let $h_{\lambda, t}$ be the Hessian of $E_{\lambda, t}$ at $\theta(\lambda, t)$. As before, $h_{\lambda, t}$ defines a generalized family of Fredholm quadratic forms $h: \theta^{*} T F(\pi) \rightarrow \mathbb{R}$. Let $\bar{h}$ be the restriction of this family to the boundary $\partial T$ of $T$. Trivializing $\theta^{*} T F(\pi)_{\mid \partial T}$ and using [19], Poposition 3.6, we conclude that $\operatorname{sf}(\bar{h})=0$. On the other hand, the spectral flow is the sum of the spectral flows of each segment of the boundary of $T$. By definition of $h_{\lambda, t}$ we have that $h_{\lambda, 0}$ is nondegenerate for all $\lambda$. Indeed, $\theta(\lambda, 0) \equiv p$ is a constant path. A $H^{1}$-vector field $\xi$ along $p$ is simply a path $\xi \in H^{1}\left(I ; T_{p}(M)\right)$ and hence $h_{0}(\xi)=d_{p}(\xi)=\int_{0}^{1} g\left(\frac{D}{d s} \xi(x), \frac{D}{d s} \xi(x)\right) \mathrm{d} x$ which is clearly nondegenerate.

From the above discussion we have that

$$
\operatorname{sf}\left(h_{(\cdot, a)},[0,1]\right)-\operatorname{sf}\left(h_{(1, \cdot)},[a, b]\right)=\operatorname{sf}\left(h_{(\cdot, b)},[0,1]\right)
$$

that is $\operatorname{sf}(h)=\mu\left(\sigma_{a}\right)-\mu\left(\sigma_{b}\right)$. 


\section{Proof of TheOrem 7.1}

Let $\gamma_{1}, \gamma_{2}$ as in (7.1). By (3.4) the Hessian of the energy functional at $\gamma_{1}$ computed on a vector field $\xi$ along $\gamma$ is given by

$$
h_{\gamma_{1}}(\xi)=\int_{0}^{1} g_{1}\left(\frac{D}{\mathrm{~d} x} \xi(x), \frac{D}{\mathrm{~d} x} \xi(x)\right) \mathrm{d} x-\int_{0}^{1} g_{1}\left(S^{1}(x) \xi(x), \xi(x)\right) \mathrm{d} x .
$$

Denoting by $\Phi: H_{0}^{1}\left(\gamma_{1}\right) \rightarrow H_{0}^{1}\left(\gamma_{2}\right)$ the bounded operator induced by $\varphi$ we have that

$$
h_{\gamma_{2}}(\Phi \xi)=\int_{0}^{1} g_{2}\left(\frac{D}{\mathrm{~d} x} \varphi_{x} \xi(x), \frac{D}{\mathrm{~d} x} \varphi_{x} \xi(x)\right) \mathrm{d} x-\int_{0}^{1} g_{2}\left(S^{2}(x) \varphi_{x} \xi(x), \varphi_{x} \xi(x)\right) \mathrm{d} x .
$$

Since parallel translation commutes with $\frac{D}{\mathrm{~d} x}$ and $\varphi_{0}$ is an isometry it follows that

$$
g\left(\frac{D}{\mathrm{~d} x} \xi(x), \frac{D}{\mathrm{~d} x} \xi(x)\right)=g\left(\frac{D}{\mathrm{~d} x} \varphi_{x} \xi(x), \frac{D}{\mathrm{~d} x} \varphi_{x} \xi(x)\right)
$$

pointwise and hence the first two integrals in (9.1), (9.2) coincide. Therefore the inequality

$$
g\left(S^{2}(x) \varphi_{x} \xi(x), \varphi_{x} \xi(x)\right) \leq g\left(S^{1}(x) \xi(x), \xi(x)\right)
$$

implies that $h_{\gamma_{2}}(\Phi \xi) \leq h_{\gamma_{1}}(\xi)$, for all $\xi \in H_{0}^{1}\left(\gamma_{1}\right)$.

Let $\tilde{\gamma}^{i}, i=1,2$ be the canonical paths induced by $\gamma_{i}$ on $\Omega\left(M_{i}\right)$ and let $h_{\lambda}$ be the Hessian of $E_{\lambda}$ at $\tilde{\gamma}_{\lambda}^{i}$.

Since the top order terms (those containing $\frac{D}{\mathrm{~d} x}$ ) of $h_{\lambda}^{1}$ and $h_{\lambda}^{2} \Phi_{\lambda}$ coincide, the difference $h_{\lambda}^{1}-h_{\lambda}^{2} \Phi_{\lambda}$ is weakly semi-continuous. By the proof of Proposition 8.4

$$
h_{0}^{1}(\xi)=\int_{0}^{1} g_{1}\left(\frac{D}{\mathrm{~d} x} \xi, \frac{D}{\mathrm{~d} x} \xi\right) \mathrm{d} x=\int_{0}^{1} g_{2}\left(\frac{D}{\mathrm{~d} x} \varphi_{0} \xi, \frac{D}{\mathrm{~d} x} \varphi_{0} \xi\right) \mathrm{d} x=h_{0}^{2}\left(\Phi_{0} \xi\right) .
$$

Moreover by hypothesis

$$
h_{1}^{2}\left(\Phi_{1} \xi\right)=h_{\gamma_{2}}(\Phi \xi) \leq h_{\gamma_{1}}(\xi)=h_{1}^{1}(\xi) .
$$

On the other hand, $\mu\left(\gamma_{1}\right)=-\operatorname{sf}\left(h^{1}\right)$ while $\mu\left(\gamma_{2}\right)=-\operatorname{sf}\left(h^{2}\right)=-\operatorname{sf}\left(h^{2} \Phi\right)$.

Taking in account (9.3) and (9.4), in order to conclude the proof of the comparison theorem it is enough to show that if $h$ and $k$ are two admissible generalized families of Fredholm quadratic forms on a Hilbert bundle $\mathcal{H}$ over $[a, b]$ whose difference is weakly semi-continuous and such that $h_{a}=k_{a}, k_{b} \leq h_{b}$, then $\operatorname{sf}(k) \leq \operatorname{sf}(h)$. The rest of the proof is devoted to show this.

Without loss of generality we can assume that $\mathcal{H}=[a, b] \times H$ is trivial. Since $h_{\lambda}-k_{\lambda}$ is weakly compact it follows that $q(s, \lambda)=s h_{\lambda}+(1-s) k_{\lambda}$, is a Fredholm quadratic form for all $(s, \lambda) \in[0,1] \times[a, b]$. The compactness of $T=[0,1] \times[a, b]$ together with the property that the space of Fredholm quadratic forms is open imply that there exists a small $\eta>0$ such that $q(s, \lambda)+t|| \cdot \|^{2}$ is still Fredholm, for any $(s, \lambda) \in T$ and $|t| \leq \eta$. Taking possibly a smaller $\eta$ we can further assume that $h_{i}+t|| \cdot \|^{2}$ is non degenerate for $i=a, b$ and $|t| \leq \eta$.

Define $\tilde{h}_{\lambda}=h_{\lambda}+\lambda \eta\|\cdot\|^{2}$. Then $\tilde{h}$ is a family of Fredholm quadratic forms that is homotopic to $h$ by the admissible homotopy $(t, \lambda) \rightarrow h_{\lambda}+t \lambda \eta\|\cdot\|^{2}$ and therefore $\operatorname{sf}(\tilde{h})=\operatorname{sf}(h)$. But now $\tilde{h}_{0}=k_{0}$ and $k_{1}<\tilde{h}_{1}$.

Consider the family of Fredholm quadratic forms defined on $T$ by $\phi(s, \lambda)=s \tilde{h}_{\lambda}+(1-s) k_{\lambda}$. Exactly as in the proof of the main theorem, the closed path obtained by restricting $\phi$ to $\partial T$ has spectral flow zero. On the other hand, its spectral flow is the sum of the spectral flows of each segment of the boundary of $[0,1] \times[a, b]$ and by the same arguments as in the proof of Theorem 4.2, we have: $\operatorname{sf}(\tilde{h})-\operatorname{sf}(k)=\operatorname{sf}(\rho)$, where $\rho(s)=(1-s) k_{1}+s \tilde{h}_{1}$.

Since $\dot{\rho}=h_{1}-k_{1}$ is positive definite if $\Sigma(\rho)$ is non empty all points $s \in \Sigma(\rho)$ are regular and, by Remark 8.3, each gives a positive contribute $\operatorname{dim} \operatorname{ker} \rho(s)$ to the spectral flow of $\rho$. Thus $\operatorname{sf}(\rho) \geq 0$ and hence $\operatorname{sf}(k) \leq \operatorname{sf}(h)$. 
In our comparison theorem it is possible to avoid the non degeneracy condition by introducing the concept of extended generalized Morse index analogous to the extended Morse index (index plus nullity) in the classical case (compare with $[9,14,21,43])$.

Let $h$ be a generalized family of Fredholm quadratic forms on a Hilbert bundle $\mathcal{H}$ over $[a, b]$. Let us consider the self-adjoint Fredholm operators $L_{\lambda}$ representing $h_{\lambda}$ with respect to the scalar product. Self-adjoint Fredholm operators are characterized by the fact that 0 is an isolated point in the spectrum of finite multiplicity. Thus, there exists a number $\delta_{0}>0$ so that $h_{\lambda}+\delta\|\cdot\|^{2}=1 / 2\langle(L+\delta) u, u\rangle$ is not degenerate for $\lambda=a, b$ and for any $0<\delta \leq \delta_{0}$. Since the set of all Fredholm quadratic forms is open in the set of all quadratic forms, $\delta_{0}$ can be chosen in such a way that $h_{\lambda}+\delta\|\cdot\|^{2}$ is a Fredholm form for $\lambda \in[a, b]$ and $0<\delta \leq \delta_{0}$.

The extended spectral flow of the family $h$ is

$$
\overline{\operatorname{sf}}(h)=\operatorname{sf}\left(h_{\lambda}+\delta\|\cdot\|^{2}, \lambda \in[a, b]\right) .
$$

for small enough $\delta$.

The normalization property for the spectral flow implies that the right hand side does not depend on the choice of $\delta$. The extended spectral flow is clearly additive under concatenation and direct sum. It is homotopy invariant under homotopies keeping the end points fixed.

The extended (generalized) Morse index is defined for any $p$-geodesic by

$$
\bar{\mu}(\gamma)=-\overline{\mathrm{sf}}(h)
$$

where $h$ is the family of hessians at the canonical path $\tilde{\gamma}$.

Arguing as above with the perturbed families we obtain:

Theorem 9.1. If $\gamma_{1}, \gamma_{2}$ are two (possibly degenerate) p-geodesics as in Theorem 7.1 , related by (7.3) then

$$
\bar{\mu}\left(\gamma_{2}\right) \leq \bar{\mu}\left(\gamma_{1}\right)
$$

\section{Appendix A. The vector Bundle neighborhood Theorem}

This section is devoted to a technical result, which is used in the proof of the main theorem.

Theorem A.1. Let $E$ and $B$ be two $C^{\infty}$ Banach manifolds such that $E$ admits a $C^{\infty}$ partition of unity and $B$ is compact. Let $\pi: E \rightarrow B$ be a $C^{\infty}$ split submersion, i.e. the differential is surjective and the kernel splits. Let $\tau: B \rightarrow E$ be a smooth section of $\pi$. Then there exist a Banach bundle $\pi^{\prime}: F \rightarrow B$, an open neighborhood $V$ of the 0-section in $F$ and a fiber preserving diffeomorphism $j: V \hookrightarrow U$, of $V$ with an open neighborhood $U$ of $\tau(B)$ such that $\tau(b)=j\left(0_{b}\right)$ for all $b \in B$.

The proof of this result, follows the construction of tubular neighborhoods of submanifolds based on sprays. However here we need sprays whose exponential map is fiber preserving. The construction is that of Palais in [37], see also [13].

Let $\pi: E \rightarrow B$ be a split submersion and let $p: T F(\pi) \rightarrow E$ be the associated bundle of tangents along the fibers. A $C^{\infty}$-vector field $\xi$ on $T F(\pi)$ is called a bundle spray over $E$ if it satisfies the following two conditions:

(1) $T p(\xi(v))=v \quad \forall v \in T F(\pi)$;

(2) $\xi(s v)=T \tilde{s}(s \xi(v)) \quad \forall s \in \mathbb{R} \quad \forall v \in T F(\pi)$

where $\tilde{s}: T F(\pi) \rightarrow T F(\pi)$ is the multiplication map given by $v \mapsto s v$ for any $v \in T F(\pi)$.

Condition (1) implies that for all $v \in T\left(E_{b}\right)$, also $T p(\xi(v)) \in T\left(E_{b}\right)$ and then $\xi(v) \in T\left(T\left(E_{b}\right)\right)$. Therefore, if $u:[0,1] \rightarrow T F(\pi)$ is an integral curve of $\xi$ with $u\left(t_{0}\right) \in T\left(E_{b}\right)$, then $u([0,1]) \subset T\left(E_{b}\right)$ and so $p u([0,1]) \subset E_{b}$.

Proof. The existence of a bundle spray $\xi$ over $E$ follows from the implicit function theorem and partition of unity arguments. Indeed, let $e \in E$ be a point. Since $\pi: E \rightarrow B$ is a split submersion, by the implicit function theorem, there exists a neighborhood $U_{e}$ of $e$ in $E$ and two open sets $V_{b} \subset B$ and $W_{b} \subset \pi^{-1}(b)$ for some $b \in B$ 
such that $U_{e}=V_{b} \times W_{b}$. Now let us consider the vertical tangent bundle over the open set $U_{e}$. By definition, we have

$$
T F\left(U_{e}\right)=T F\left(V_{b} \times W_{b}\right)=V_{b} \times T\left(W_{b}\right) .
$$

In this way we are able to construct a bundle spray locally on $T F\left(U_{e}\right)$ extending to the product an ordinary spray on $T\left(W_{b}\right)$ in the obvious way. Observing that a convex combination of two bundle sprays is again a bundle spray, we can take a smooth partition of unity on $E$ and glue the local sprays in order to get a bundle spray on $T F(\pi)$.

If $\xi$ is a bundle spray for $\pi$, the associated exponential map, is defined as follows: for each $v \in T F(E)$ let $\sigma_{v}$ be the maximal integral curve of $\xi$ such that $\sigma_{v}(0)=v$. The second condition for a bundle spray implies that there exists an open neighborhood $\mathcal{D}$ of the zero section in $T F(E)$ such that for all $v \in \mathcal{D}, \sigma_{v}(1)$ is defined. Now by definition $\exp (v)=p\left(\sigma_{v}(1)\right) \quad \forall v \in \mathcal{D}$.

Let $F=\tau^{*}(T F)$. If $i: F \rightarrow T F$ is the canonical inclusion and $V=i^{-1}(\mathcal{D})$ we define $j: V \rightarrow E$ by $j(v)=\exp i(v)$. By construction of the exponential map $j$ is fiber preserving and clearly $j\left(0_{b}\right)=\tau(b)$. We want to show that $j$ restricted to a (possibly) smaller neighborhood $V$ of the zero section is a diffeomorphism. For this, if $j_{b}: F_{b} \rightarrow E_{b}$ is the restriction of $j$ to the fiber, identifying $F_{b}$ with its tangent space in the canonical way and arguing as in [32], Theorem 4.1, we show that $T j_{b}\left(0_{b}\right)=i d$. Since $j$ is fiber preserving this easily implies that $T j\left(0_{b}\right)$ is injective and hence invertible since, being $B$ finite dimensional, $T j\left(0_{b}\right)$ is Fredholm of index 0 . Thus $j$ is a local diffeomorphism. Since this holds for every $b \in B$, by a standard compactness argument there must be a (possibly) smaller neighborhood $V$ of the zero section such that the restriction of $j$ to that neighborhood is a (fiber preserving) diffeomorphism with a neighborhood $U$ of $\tau(B)$. This concludes the proof.

Acknowledgements. This work has been partly supported by grant Fondecyt 1040936, Chile.

\section{REFERENCES}

[1] R. Abraham and J.E. Marsden, Foundations of Mechanics, 2nd edition. Benjamin/Cummings, Ink. Massachusetts (1978).

[2] L. Andersson and R. Howard, Comparison and rigidity theorems in Semi-Riemannian geometry. Comm. Anal. Geom. 6 (1998) 819-877.

[3] S.B. Angenent and R. van der Vorst, A priori bounds and renormalized Morse indices of solutions of an elliptic system. Ann. Inst. H. Poincaré Anal. Non Linéaire 17 (2000) 277-306.

[4] V.I. Arnol'd, Sturm theorems and symplectic geometry. Funktsional. Anal. i Prilozhen. 19 (1985) 1-10.

[5] J.K. Beem, P.E. Ehrlich and K.L. Easley, Global Lorentzian Geometry. Mercel Dekker, Inc. New York and Basel (1996).

[6] V. Benci, F. Giannoni and A. Masiello, Some properties of the spectral flow in semiriemannian geometry. J. Geom. Phys. 27 (1998) 267-280.

[7] A.L. Besse, Manifolds all of whose geodesics are closed. Ergebnisse der Mathematik und ihrer Grenzgebiete 93, Springer-Verlag (1978).

[8] O. Bolza, Lectures on Calculus of Variation. Univ. Chicago Press, Chicago (1904).

[9] S.E. Cappell, R. Lee and E.Y. Miller, On the Maslov index. Comm. Pure Appl. Math. 47 (1994) 121-186.

[10] I. Chavel, Riemannian geometry: a modern introduction, in Cambridge tracts in Mathematics 108, Cambridge Univerisity Press (1993).

[11] P. Chossat, D. Lewis, J.P. Ortega and T.S. Ratiu, Bifurcation of relative equilibria in mechanical systems with symmetry. Adv. Appl. Math. 31 (2003) 10-45.

[12] C. Conley and E. Zehnder, The Birhoff-Lewis fixed point theorem and a conjecture of V.I. Arnold. Invent. Math. 73 (1983) 33-49.

[13] M. Crabb and I. James, Fibrewise Homotopy Theory. Springer-Verlag (1998).

[14] M. Daniel, An extension of a theorem of Nicolaescu on spectral flow and Maslov index. Proc. Amer. Math. Soc. 128 (1999) 611-619.

[15] K. Deimling, Nonlinear Functional Analysis. Springer-Verlag (1985).

[16] I. Ekeland, Convexity methods in Hamiltonian systems. Ergebnisse der Mathematik und ihrer Grenzgebiete 19, SpringerVerlag, Berlin (1990).

[17] Guihua Fei, Relative Morse index and its application to Hamiltonian systems in the presence of symmetries. J. Diff. Eq. 122 (1995) 302-315.

[18] P.M. Fitzpatrick and J. Pejsachowicz, Parity and generalized multiplicity. Trans. Amer. Math. Soc. 326 (1991) $281-305$. 
[19] P.M. Fitzpatrick, J. Pejsachowicz and L. Recht, Spectral flow and bifurcation of critical points of strongly-indefinite functional. Part I. General theory. J. Funct. Anal. 162 (1999) 52-95.

[20] P.M. Fitzpatrick, J. Pejsachowicz and L. Recht, Spectral flow and bifurcation of critical points of strongly-indefinite functional. Part II. Bifurcation of periodic orbits of Hamiltonian systems. J. Differ. Eq. 161 (2000) 18-40.

[21] A. Floer, Relative Morse index for the symplectic action. Comm. Pure Appl. Math. 41 (1989) 335-356.

[22] I.M. Gel'fand and S.V. Fomin, Calculus of Variations. Prentic-Hall Inc., Englewood Cliffs, New Jersey, USA (1963).

[23] I.M. Gel'fand and V.B. Lidskii, On the structure of the regions of stability of linear canonical systems of differential equations with periodic coefficients. Amer. Math. Soc. Transl. Ser. 28 (1958) 143-181.

[24] R. Giambó, P. Piccione and A. Portaluri, On the Maslov Index of Lagrangian paths that are not transversal to the Maslov cycle. Semi-Riemannian index Theorems in the degenerate case. (2003) Preprint.

[25] A.D. Helfer, Conjugate points on space like geodesics or pseudo self-adjoint Morse-Sturm-Liouville systems. Pacific J. Math. 164 (1994) 321-340.

[26] J. Jost, X. Li-Jost and X.W. Peng, Bifurcation of minimal surfaces in Riemannian manifolds. Trans. Amer. Math. Soc. 347 (1995) 51-62.

[27] T. Kato, Perturbation Theory for linear operators. Grundlehren der Mathematischen Wissenschaften 132, Springer-Verlag (1980).

[28] W. Klingenberg, Closed geodesics on Riemannian manifolds. CBMS Regional Conference Series in Mathematics 53 (1983).

[29] W. Klingenberg, Riemannian Geometry. de Gruyter, New York (1995).

[30] M.A. Krasnoselskii, Topological methods in the theory of nonlinear integral equations. Pergamon, New York (1964).

[31] D.N. Kupeli, On conjugate and focal points in semi-Riemannian geometry. Math. Z. 198 (1988) 569-589.

[32] S. Lang, Differential and Riemannian Manifolds. Springer-Verlag (1995).

[33] E. Meinrenken, Trace formulas and Conley-Zehnder index. J. Geom. Phys. 13 (1994) 1-15.

[34] J. Milnor, Morse theory. Based on lecture notes by M. Spivak and R. Wells. Annals of Mathematics Studies 51, Princeton University Press, Princeton, N.J. (1963).

[35] M. Musso, J. Pejsachowicz and A. Portaluri, A Morse Index Theorem and bifurcation for perturbed geodesics on SemiRiemannian Manifolds. Topol. Methods Nonlinear Anal. 25 (2005) 69-99.

[36] B. O'Neill, Semi-Riemannian geometry with applications to relativity. Academic Press, New York (1983).

[37] R.S. Palais, Foundations of global non-linear analysis. W.A. Benjamin, Inc., New York (1968).

[38] G. Peano, Lezioni di Analisi infinitesimale, Volume I, pp. 120-121, Volume II, pp. 187-195. Tipografia editrice G. Candeletti, Torino (1893).

[39] P. Piccione, A. Portaluri and D.V. Tausk, Spectral flow, Maslov index and bifurcation of semi-Riemannian geodesics. Ann. Global Anal. Geometry 25 (2004) 121-149.

[40] A. Portaluri, A formula for the Maslov index of linear autonomous Hamiltonian systems. (2004) Preprint.

[41] A. Portaluri, Morse Index Theorem and Bifurcation theory on semi-Riemannian manifolds. Ph.D. thesis (2004).

[42] P.J. Rabier, Generalized Jordan chains and two bifurcation theorems of Krasnosel'skii. Nonlinear Anal. 13 (1989) 903-934.

[43] J. Robbin and D. Salamon, The Maslov index for paths. Topology 32 (1993) 827-844.

[44] J. Robbin and D. Salamon, The spectral flow and the Maslov index. Bull. London Math. Soc. 27 (1995) 1-33. 